\title{
Some Explicit Expressions and Interesting Bifurcation Phenomena for Nonlinear Waves in Generalized Zakharov Equations
}

\author{
Shaoyong $\mathrm{Li}^{1,2}$ and Rui Liu ${ }^{1}$ \\ ${ }^{1}$ Department of Mathematics, South China University of Technology, Guangzhou, Guangdong 510640, China \\ ${ }^{2}$ College of Mathematics and Information Sciences, Shaoguan University, Shaoguan, Guangdong 512005, China \\ Correspondence should be addressed to Rui Liu; scliurui@scut.edu.cn
}

Received 22 December 2012; Revised 7 February 2013; Accepted 17 February 2013

Academic Editor: Chun-Lei Tang

Copyright (C) 2013 S. Li and R. Liu. This is an open access article distributed under the Creative Commons Attribution License, which permits unrestricted use, distribution, and reproduction in any medium, provided the original work is properly cited.

Using bifurcation method of dynamical systems, we investigate the nonlinear waves for the generalized Zakharov equations $u_{t t}-$ $c_{s}^{2} u_{x x}=\beta\left(|E|^{2}\right)_{x x}, i E_{t}+\alpha E_{x x}-\delta_{1} u E+\delta_{2}|E|^{2} E+\delta_{3}|E|^{4} E=0$, where $\alpha, \beta, \delta_{1}, \delta_{2}, \delta_{3}$, and $c_{s}$ are real parameters, $E=E(x, t)$ is a complex function, and $u=u(x, t)$ is a real function. We obtain the following results. (i) Three types of explicit expressions of nonlinear waves are obtained, that is, the fractional expressions, the trigonometric expressions, and the exp-function expressions. (ii) Under different parameter conditions, these expressions represent symmetric and antisymmetric solitary waves, kink and antikink waves, symmetric periodic and periodic-blow-up waves, and 1-blow-up and 2-blow-up waves. We point out that there are two sets of kink waves which are called tall-kink waves and low-kink waves, respectively. (iii) Five kinds of interesting bifurcation phenomena are revealed. The first kind is that the 1-blow-up waves can be bifurcated from the periodic-blow-up and 2-blow-up waves. The second kind is that the 2-blow-up waves can be bifurcated from the periodic-blow-up waves. The third kind is that the symmetric solitary waves can be bifurcated from the symmetric periodic waves. The fourth kind is that the low-kink waves can be bifurcated from four types of nonlinear waves, the symmetric solitary waves, the 1-blow-up waves, the tall-kink waves, and the antisymmetric solitary waves. The fifth kind is that the tall-kink waves can be bifurcated from the symmetric periodic waves. We also show that the exp-function expressions include some results given by pioneers.

\section{Introduction}

Since the exact solutions to nonlinear wave equations help to understand the characteristics of nonlinear equations, seeking exact solutions of nonlinear equations is an important subject. For this purpose, there have been many methods such as the Jacobi elliptic function method $[1,2]$, F-expansion method $[3,4]$, and $\left(G^{\prime} / G\right)$-expansion method $[5,6]$.

Recently, the bifurcation method of dynamical systems [7-9] has been introduced to study the nonlinear partial differential equations. Up to now, the method is widely used in literatures such as [10-16].

In this paper, we consider the generalized Zakharov equations [17], which read as

$$
\begin{gathered}
u_{t t}-c_{s}^{2} u_{x x}=\beta\left(|E|^{2}\right)_{x x}, \\
i E_{t}+\alpha E_{x x}-\delta_{1} u E+\delta_{2}|E|^{2} E+\delta_{3}|E|^{4} E=0,
\end{gathered}
$$

where $\alpha, \beta, \delta_{1}, \delta_{2}, \delta_{3}$, and $c_{s}$ are real parameters. $E=E(x, t)$ is a complex function which represents the envelop of the electric field, and $u=u(x, t)$ is a real function which represents the plasma density measured from its equilibrium value. Huang and Zhang [17] used Fan's direct algebraic method to obtain some exact travelling wave solutions of (1) as follows:

$$
\begin{array}{ll}
u_{a 0}^{ \pm}=\frac{\beta}{c^{2}-c_{s}^{2}}\left(\varphi_{a 0}^{ \pm}\right)^{2}, & E_{a 0}^{ \pm}=\varphi_{a 0}^{ \pm} e^{i(\gamma x-\omega t)}, \\
u_{b 0}^{ \pm}=\frac{\beta}{c^{2}-c_{s}^{2}}\left(\varphi_{b 0}^{ \pm}\right)^{2}, & E_{b 0}^{ \pm}=\varphi_{b 0}^{ \pm} e^{i(\gamma x-\omega t)}, \\
u_{c 0}^{ \pm}=\frac{\beta}{c^{2}-c_{s}^{2}}\left(\varphi_{c 0}^{ \pm}\right)^{2}, & E_{c 0}^{ \pm}=\varphi_{c 0}^{ \pm} e^{i(\gamma x-\omega t)},
\end{array}
$$




$$
\begin{array}{ll}
u_{d 0}^{ \pm}=\frac{\beta}{c^{2}-c_{s}^{2}}\left(\varphi_{d 0}^{ \pm}\right)^{2}, & E_{d 0}^{ \pm}=\varphi_{d 0}^{ \pm} e^{i(\gamma x-\omega t)}, \\
u_{e 0}^{ \pm}=\frac{\beta}{c^{2}-c_{s}^{2}}\left(\varphi_{e 0}^{ \pm}\right)^{2}, & E_{e 0}^{ \pm}=\varphi_{e 0}^{ \pm} e^{i(\gamma x-\omega t)},
\end{array}
$$

where $\gamma$ and $w$ are two constants and

$$
\begin{aligned}
& \varphi_{a 0}^{ \pm}= \pm \sqrt{\frac{-12 p}{3 q-\sqrt{9 q^{2}-48 p r} \cos (2 \sqrt{-p} \xi)}}, \\
& \varphi_{b 0}^{ \pm}= \pm \sqrt{\frac{2 p}{q}(-1+\tanh (\sqrt{p} \xi))}, \\
& \varphi_{c 0}^{ \pm}= \pm \sqrt{\frac{2 p}{q}(-1-\tanh (\sqrt{p} \xi))} \text {, } \\
& \varphi_{d 0}^{ \pm}= \pm \sqrt{\frac{12 p}{-3 q+\sqrt{9 q^{2}-48 p r} \cosh (2 \sqrt{p} \xi)}}, \\
& \varphi_{e 0}^{ \pm}= \pm \sqrt{\frac{2 p}{q}(-1-\operatorname{coth}(\sqrt{p} \xi))} \text {, } \\
& \xi=x-c t, \\
& p=\frac{\alpha \gamma^{2}-\omega}{\alpha} \\
& q=\frac{\beta \delta_{1}}{\alpha\left(c^{2}-c_{s}^{2}\right)}-\frac{\delta_{2}}{\alpha}, \\
& r=-\frac{\delta_{3}}{\alpha}, \\
& c=2 \alpha \gamma .
\end{aligned}
$$

When $\alpha=1, \beta=-1, \delta_{1}=-2, \delta_{2}=2 \lambda, \delta_{3}=0$, and $c_{s}=$ 1 , (1) reduce to the equations

$$
\begin{gathered}
u_{t t}-u_{x x}+\left(|E|^{2}\right)_{x x}=0, \\
i E_{t}+E_{x x}+2 u E+2 \lambda|E|^{2} E=0 .
\end{gathered}
$$

El-Wakil et al. [18] used the extended Jacobi elliptic function expansion method to obtain some Jacobi elliptic function expression solutions of (13).

When $\lambda=0,(13)$ reduce to the equations

$$
\begin{gathered}
u_{t t}-u_{x x}+\left(|E|^{2}\right)_{x x}=0, \\
i E_{t}+E_{x x}+2 u E=0 .
\end{gathered}
$$

By multisymplectic numerical method, Wang [19] proved the preservation of discrete normal conservation law of (14) theoretically and investigated the propagation and collision behaviors of the solitary waves numerically. There are also many other researchers studying (1) or its special case; for more information, one can see [20-24].
In this paper, we investigate the nonlinear waves and the bifurcation phenomena of (1). Coincidentally, under some transformations, (1) reduce to a planar system (54) which is similar to the planar system obtained by Feng and Li [16]. Many exact explicit parametric representations of solitary waves, kink and antikink waves, and periodic waves were obtained in [16], and their work is very important for the $\phi^{6}$ model. In order to find the travelling wave solutions of (1), here we consider (1) by using the bifurcation method mentioned above; firstly, we obtain three types of explicit nonlinear wave solutions, this is, the fractional expressions, the trigonometric expressions, and the exp-function expressions. Secondly, we point out that these expressions represent symmetric and antisymmetric solitary waves, kink and antikink waves, symmetric periodic and periodic-blow-up waves, and 1-blow-up and 2-blow-up waves under different parameter conditions. Thirdly, we reveal five kinds of interesting bifurcation phenomena mentioned in the abstract above.

The remainder of this paper is organized as follows. In Section 2, we give some notations and state our main results. In Section 3, we give derivations for our results. A brief conclusion is given in Section 4.

\section{Main Results}

In this section, we state our main results. To relate conveniently, let us give some notations which will be used in the latter statement and the derivations.

Let $l_{i}(i=1,2, \ldots, 7)$ represent the following seven curves:

$$
\begin{array}{cc}
l_{1}: p=\frac{q^{2}}{4 r} \quad(r>0, q<0), \\
l_{2}: p=\frac{3 q^{2}}{16 r} \quad(r>0, q<0), \\
l_{3}: p=0 \quad(r>0, q<0), \\
l_{4}: p=0 \quad(r>0, q>0), \\
l_{5}: p=0 \quad(r<0, q>0), \\
l_{6}: p=\frac{3 q^{2}}{16 r} \quad(r<0, q>0), \\
l_{7}: p=\frac{q^{2}}{4 r} \quad(r<0, q<0) .
\end{array}
$$

Let $A_{i}(i=1,2, \ldots, 12)$ represent the regions surrounded by the curves $l_{i}(i=1,2, \ldots, 7)$ and the coordinate axes (see Figure 1).

Let

$$
\begin{aligned}
u(x, t) & =\frac{\beta}{c^{2}-c_{s}^{2}} \varphi^{2}(\xi), \\
E(x, t) & =\varphi(\xi) e^{i(\gamma x-\omega t)}, \\
\Delta & =\sqrt{q^{2}-4 p r}, \\
\varphi_{1} & =\sqrt{\frac{-q+\Delta}{2 r}},
\end{aligned}
$$




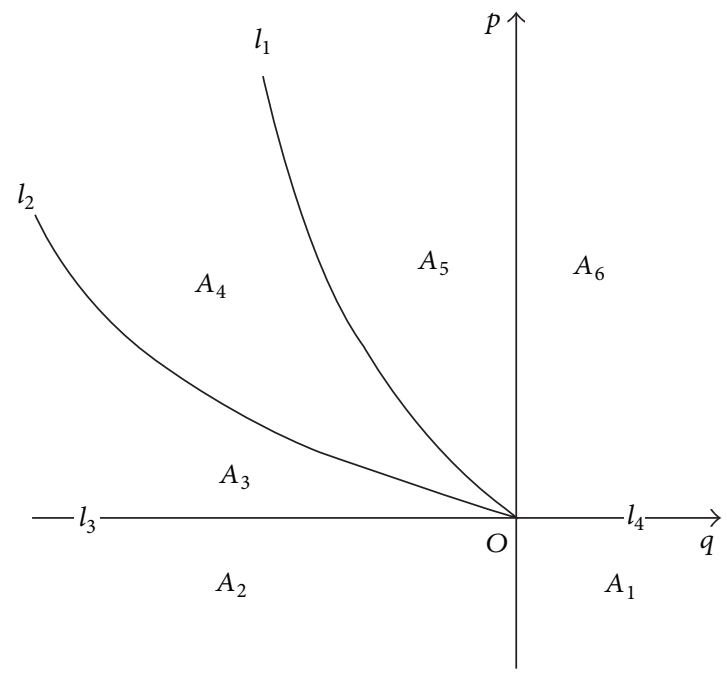

(a) $r>0$

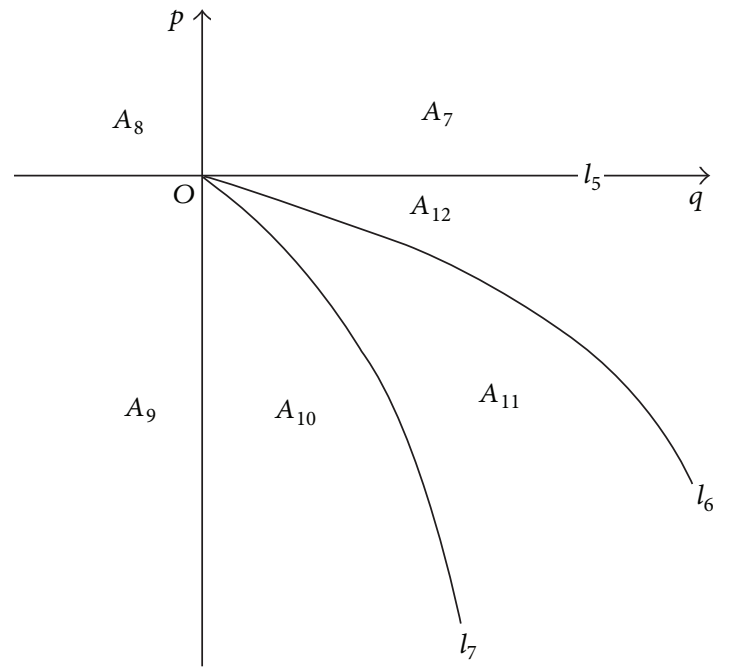

(b) $r<0$

FIgURE 1: The locations of the regions $A_{i}(i=1,2, \ldots, 12)$ and curves $l_{i}(i=1,2, \ldots, 7)$.

$$
\begin{gathered}
\varphi_{2}=\sqrt{\frac{-q-\Delta}{2 r},} \\
H(\varphi, y)=h,
\end{gathered}
$$

where $H(\varphi, y)$ is the first integral which will be given later and $h$ is the integral constant.

In order to search for the solutions of (1) and studing the bifurcation phenomena, we only need to get the solution $\varphi(\xi)$ according to (22) and (23). For convenience, throughout the following work we only discuss the solution $\varphi(\xi)$. Now let us state our main results in the following Propositions 1, 2, and 3.

\subsection{When the Orbit $\Gamma$ Is Defined by $H(\varphi, y)=H(0,0)$}

Proposition 1. (i) For $p=0$, (1) have two fractional nonlinear wave solutions

$$
u_{a}^{ \pm}=\frac{\beta}{c^{2}-c_{s}^{2}}\left(\varphi_{a}^{ \pm}\right)^{2}, \quad E_{a}^{ \pm}=\varphi_{a}^{ \pm} e^{i(\gamma x-\omega t)},
$$

where

$$
\varphi_{a}^{ \pm}= \pm \sqrt{\frac{6 q}{3 q^{2} \xi^{2}-4 r}} .
$$

If $(q, p) \in l_{4}$, then $\varphi_{a}^{ \pm}$are 1-blow-up waves (refer to Figure $2(d)$ ). If $(q, p) \in l_{3}$, then $\varphi_{a}^{ \pm}$are 2-blow-up waves (refer to Figure $3(d))$. If $(q, p) \in l_{5}$, then $\varphi_{a}^{ \pm}$are symmetric solitary waves (refer to Figure $4(d)$ ).

(ii) For $p<0$, (1) have two nonlinear wave solutions

$$
u_{b}^{ \pm}=\frac{\beta}{c^{2}-c_{s}^{2}}\left(\varphi_{b}^{ \pm}\right)^{2}, \quad E_{b}^{ \pm}=\varphi_{b}^{ \pm} e^{i(\gamma x-\omega t)},
$$

where

$$
\varphi_{b}^{ \pm}= \pm \sqrt{\frac{-12 p}{3 q+\sqrt{9 q^{2}-48 p r} \cos (2 \sqrt{-p} \xi)}} .
$$

These solutions have the following properties and wave shapes.

(1) When $(q, p) \in A_{1}$ or $A_{2}$, then $\varphi_{a 0}^{ \pm}$and $\varphi_{b}^{ \pm}$are periodicblow-up waves (refer to Figure 2(a) or Figure 3(a)). Specially, in region $A_{1}$ when $p \rightarrow 0-0$, the periodicblow-up waves $\varphi_{a 0}^{ \pm}$become 1-blow-up waves $\varphi_{a}^{ \pm}$, and for the varying process, see Figure 2, while the periodicblow-up waves $\varphi_{b}^{ \pm}$become a trivial wave $\varphi=0$. In region $A_{2}$ when $p \rightarrow 0-0$, the periodic-blow-up waves $\varphi_{b}^{ \pm}$become 2-blow-up waves $\varphi_{a}^{ \pm}$, and for the varying process, see Figure 3.

(2) When $(q, p) \in A_{12}$, then $\varphi_{a 0}^{ \pm}$and $\varphi_{b}^{ \pm}$are symmetric periodic waves (refer to Figure 5(a)). If $p \rightarrow 0-0$, the symmetric periodic waves $\varphi_{a 0}^{ \pm}$become symmetric solitary waves $\varphi_{a}^{ \pm}$, and for the varying process, see Figure 4. If $p \rightarrow 3 q^{2} / 16 r+0$, the symmetric periodic waves $\varphi_{a 0}^{ \pm}$and $\varphi_{b}^{ \pm}$become two trivial waves $\varphi=$ $\pm \sqrt{-(3 q / 4 r)}$, and for the varying process, see Figure 5.

(iii) For $p>0$, (1) have four nonlinear wave solutions

$$
\begin{array}{ll}
u_{c}^{ \pm}=\frac{\beta}{c^{2}-c_{s}^{2}}\left(\varphi_{c}^{ \pm}\right)^{2}, & E_{c}^{ \pm}=\varphi_{c}^{ \pm} e^{i(\gamma x-\omega t)}, \\
u_{d}^{ \pm}=\frac{\beta}{c^{2}-c_{s}^{2}}\left(\varphi_{d}^{ \pm}\right)^{2}, & E_{d}^{ \pm}=\varphi_{d}^{ \pm} e^{i(\gamma x-\omega t)},
\end{array}
$$

where

$$
\begin{aligned}
& \varphi_{c}^{ \pm}= \pm \sqrt{\frac{4 \lambda p}{\lambda^{2} e^{2 \sqrt{p} \xi}+\left(\lambda_{0} / 12\right) e^{-2 \sqrt{p} \xi}-\lambda q}}, \\
& \varphi_{d}^{ \pm}= \pm \sqrt{\frac{4 \lambda p}{\lambda^{2} e^{-2 \sqrt{p} \xi}+\left(\lambda_{0} / 12\right) e^{2 \sqrt{p} \xi}-\lambda q}},
\end{aligned}
$$

$\lambda$ is a nonzero arbitrary real constant and $\lambda_{0}=3 q^{2}-16 p r$. Corresponding to $\lambda>0$ or $\lambda<0$, these solutions have the following properties and wave shapes. 


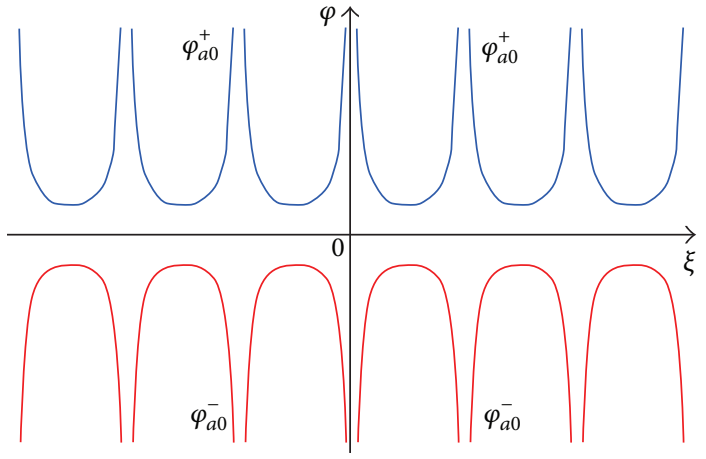

(a)

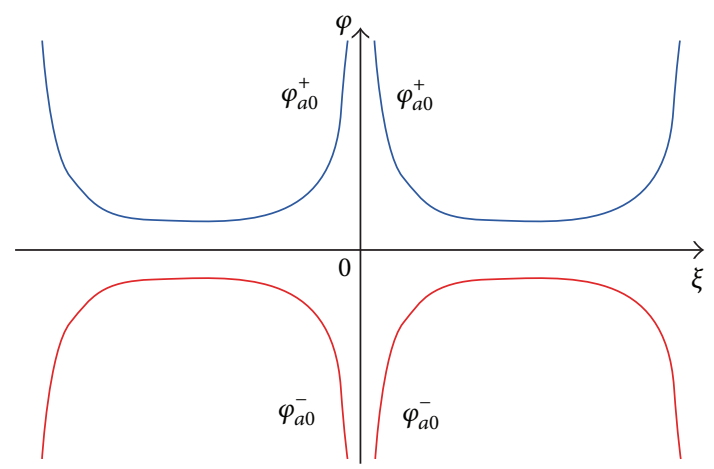

(c)

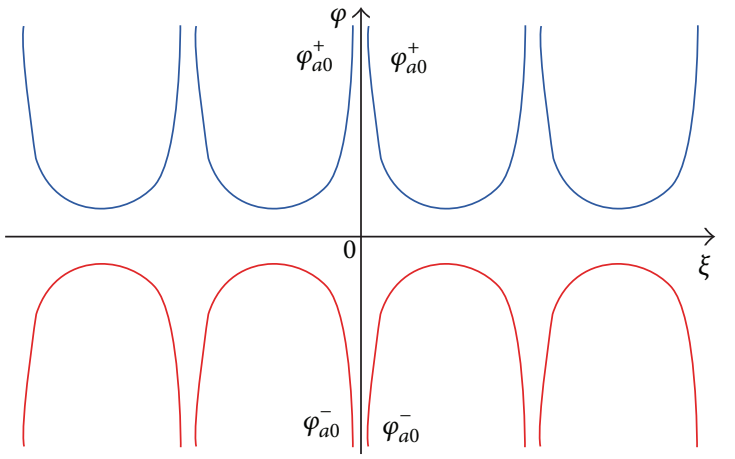

(b)

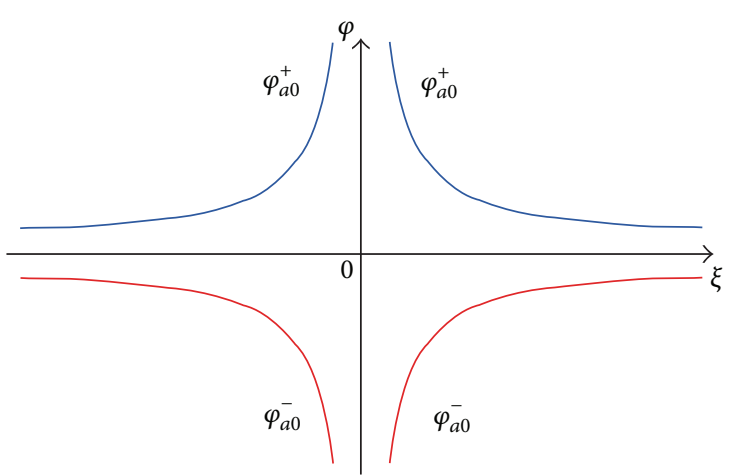

(d)

Figure 2: (The 1-blow-up waves are bifurcated from the periodic-blow-up waves.) The varying process for the figures of $\varphi_{a 0}^{ \pm}$when $(q, p) \in A_{1}$ and $p \rightarrow 0-0$, where $r=1, q=4$ and (a) $p=0-10^{-1}$, (b) $p=0-10^{-2}$, (c) $p=0-10^{-3}$, and (d) $p=0-10^{-4}$.

(1) For the case of $\lambda>0$, there are four properties as follows.

(1) When $(q, p) \in l_{2}$, that is, $r>0, q<0$, and $r=3 q^{2} / 16 p$, then $\varphi_{c}^{ \pm}$and $\varphi_{d}^{ \pm}$become

$$
\begin{gathered}
\varphi_{c 1}^{ \pm}= \pm \sqrt{\frac{4 p}{\lambda e^{2 \sqrt{p} \xi}-q}}, \\
\varphi_{d 1}^{ \pm}= \pm \sqrt{\frac{4 p}{\lambda e^{-2 \sqrt{p} \xi}-q}},
\end{gathered}
$$

which represent four low-kink waves (refer to Figure $6(d)$ or Figure $7(d)$ ). Specially, let $\lambda=$ $-q>0$, then $\varphi_{c 1}^{ \pm}$and $\varphi_{d 1}^{ \pm}$become $\varphi_{b 0}^{ \pm}$and $\varphi_{c 0}^{ \pm}$.

$(1)_{\mathrm{b}}$ If $(q, p)$ belongs to one of $A_{3}, A_{7}, A_{8}$, and $\lambda \neq \sqrt{\lambda_{0} / 12}$, then $\varphi_{c}^{ \pm} \neq \varphi_{d}^{ \pm}$, and they represent four symmetric solitary waves (refer to Figure 6(a)). Specially, when $(q, p) \in A_{3}$ and $p \rightarrow 3 q^{2} / 16 r-0$, then the four symmetric solitary waves $\varphi_{c}^{ \pm}$and $\varphi_{d}^{ \pm}$become the four lowkink waves $\varphi_{c 1}^{ \pm}$and $\varphi_{d 1}^{ \pm}$, and for the varying process, see Figure 6.

(1) If $(q, p)$ belongs to one of $A_{4}, A_{5}, A_{6}, l_{1}$, and $\lambda \neq \sqrt{\lambda_{0} / 12}$, then $\varphi_{c}^{ \pm} \neq \varphi_{d}^{ \pm}$, and they represent four 1-blow-up waves (refer to Figure 7(a)). Specially, when $(q, p) \in A_{4}$ and $p \rightarrow 3 q^{2} / 16 r+0$, then the four 1-blow-up waves $\varphi_{c}^{ \pm}$and $\varphi_{d}^{ \pm}$become the four low-kink waves $\varphi_{c 1}^{ \pm}$and $\varphi_{d 1}^{ \pm}$, and for the varying process, see Figure 7.

$(1)_{\mathrm{d}}$ If $(q, p)$ belongs to one of $A_{3}, A_{7}, A_{8}$, and $\lambda=$ $\sqrt{\lambda_{0} / 12}$, then $\varphi_{c}^{ \pm}=\varphi_{d}^{ \pm}=\varphi_{d 0}^{ \pm}$. Specially, when $(q, p) \in A_{3}$ and $p \rightarrow 3 q^{2} / 16 r-0$, then $\varphi_{d 0}^{ \pm}$tend to two trivial solutions $\varphi= \pm \sqrt{-(3 q / 4 r)}$.

(2) For the case of $\lambda<0$, there are three properties as follows.

(2) $)_{\mathrm{a}}$ If $(q, p) \in l_{2}$, that is, $r>0, q<0$, and $r=3 q^{2} / 16 p$, then $\varphi_{c}^{ \pm}$and $\varphi_{d}^{ \pm}$become $\varphi_{c 1}^{ \pm}$and $\varphi_{d 1}^{ \pm}$which represent four 1-blow-up waves (refer to Figure $8(d))$. Specially, let $\lambda=q<0$, then $\varphi_{c 1}^{ \pm}$ and $\varphi_{d 1}^{ \pm}$become the hyperbolic 1-blow-up wave solutions $\varphi_{e 0}^{ \pm}$and

$$
\varphi_{d 2}^{ \pm}= \pm \sqrt{\frac{2 p}{q}(-1+\operatorname{coth}(\sqrt{p} \xi))} .
$$

$(2)_{\mathrm{b}}$ If $(q, p) \in A_{3}$ and $\lambda \neq-\sqrt{\lambda_{0} / 12}$, then $\varphi_{c}^{ \pm} \neq \varphi_{d}^{ \pm}$, and they represent four 2-blow-up waves (refer to Figure 8(a)). Specially, when $p \rightarrow 3 q^{2} / 16 r-0$, then the four 2-blow-up waves become four 1blow-up waves $\varphi_{c 1}^{ \pm}$and $\varphi_{d 1}^{ \pm}$, and for the varying process, see Figure 8. 


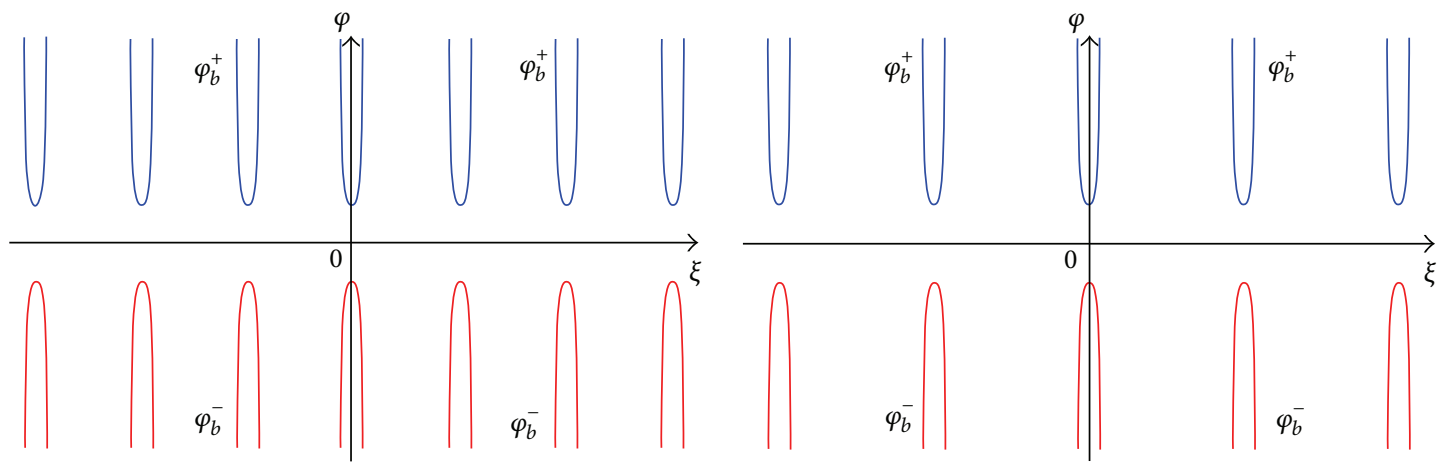

(a)

(b)
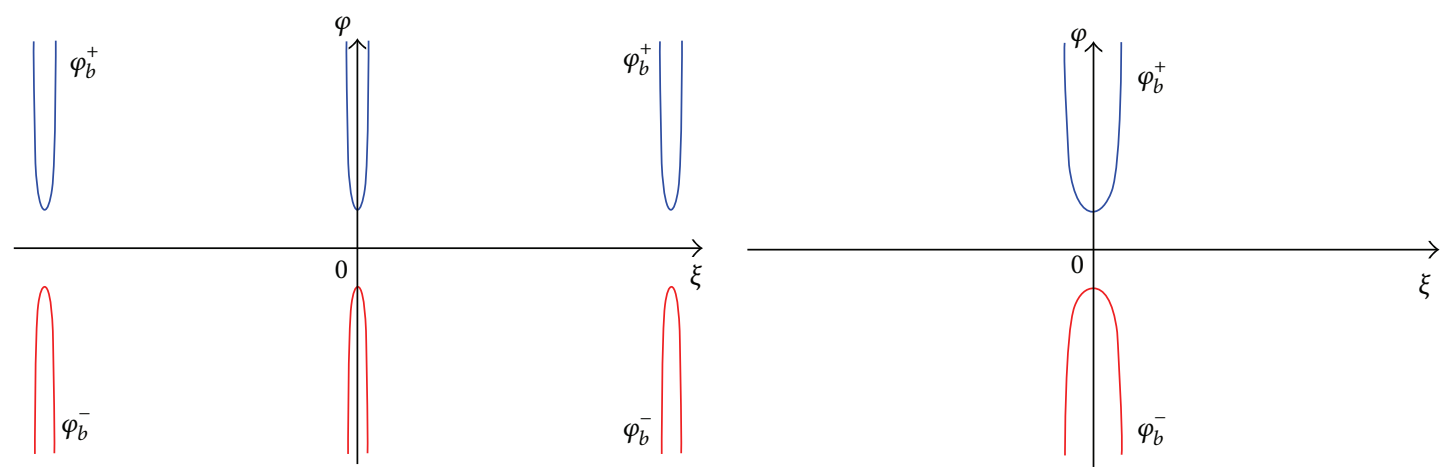

(c)

(d)

Figure 3: (The 2-blow-up waves are bifurcated from the periodic-blow-up waves.) The varying process for the figures of $\varphi_{b}^{ \pm}$when $(q, p) \in A_{2}$ and $p \rightarrow 0-0$, where $r=1, q=-4$ and (a) $p=0-1$, (b) $p=0-0.5$, (c) $p=0-0.1$, and (d) $p=0-0.01$.

(2) If $(q, p) \in A_{3}$ and $\lambda=-\sqrt{\lambda_{0} / 12}$, then $\varphi_{c}^{ \pm}=$ $\varphi_{d}^{ \pm}=\varphi_{c d}^{ \pm}$of forms

$$
\varphi_{c d}^{ \pm}= \pm \sqrt{\frac{12 p}{-3 q-\sqrt{9 q^{2}-48 p r} \cosh (2 \sqrt{p} \xi)}}
$$

which represent hyperbolic blow-up waves. When $p \rightarrow 3 q^{2} / 16 r-0$, then $\varphi_{c d}^{ \pm}$tend to two trivial solutions $\varphi= \pm \sqrt{-(3 q / 4 r)}$.

\subsection{When the Orbit $\Gamma$ Is Defined by $H(\varphi, y)=H\left(\varphi_{1}, 0\right)$}

Proposition 2. If $(q, p)$ belongs to one of the regions $A_{1}, A_{2}, A_{3}, A_{4}, A_{11}$, and $A_{12}$ or curves $l_{1}, l_{2}, l_{3}, l_{6}$, and $l_{7}$, then (1) have four nonlinear wave solutions

$$
\begin{array}{ll}
u_{e}^{ \pm}=\frac{\beta}{c^{2}-c_{s}^{2}}\left(\varphi_{e}^{ \pm}\right)^{2}, & E_{e}^{ \pm}=\varphi_{e}^{ \pm} e^{i(\gamma x-\omega t)}, \\
u_{f}^{ \pm}=\frac{\beta}{c^{2}-c_{s}^{2}}\left(\varphi_{f}^{ \pm}\right)^{2}, & E_{f}^{ \pm}=\varphi_{f}^{ \pm} e^{i(\gamma x-\omega t)},
\end{array}
$$

where

$$
\begin{gathered}
\varphi_{e}^{ \pm}= \pm \frac{\sqrt{((\Delta-q) / 2 r)}\left(2 \Delta+q-2 \mu r e^{\theta \xi}\right)}{\sqrt{(2 \Delta+q)^{2}+4 \mu r(4 \Delta-q) e^{\theta \xi}+4 \mu^{2} r^{2} e^{2 \theta \xi}}}, \\
\varphi_{f}^{ \pm}= \pm \frac{\sqrt{((\Delta-q) / 2 r)}\left(2 \Delta+q-2 \mu r e^{-\theta \xi}\right)}{\sqrt{(2 \Delta+q)^{2}+4 \mu r(4 \Delta-q) e^{-\theta \xi}+4 \mu^{2} r^{2} e^{-2 \theta \xi}}},
\end{gathered}
$$

$\mu$ is a nonzero arbitrary real constant, $\Delta$ is given in (24), and

$$
\theta=\sqrt{\frac{1}{r} \Delta(\Delta-q)}
$$

Let

$$
\mu_{0}=\frac{q+2 \Delta}{2 r}
$$

About $\mu_{0}$, one has the following fact:

$$
\mu_{0} \begin{cases}>0 & \text { for }(q, p) \in A_{1}, A_{2}, A_{3}, l_{3} \\ =0 & \text { for }(q, p) \in l_{2} \\ <0 & \text { for }(q, p) \in A_{4}, A_{11}, A_{12}, l_{1}, l_{6}, l_{7}\end{cases}
$$

Corresponding to $\mu>0$ and $\mu<0$, these solutions have the following properties and wave shapes. 


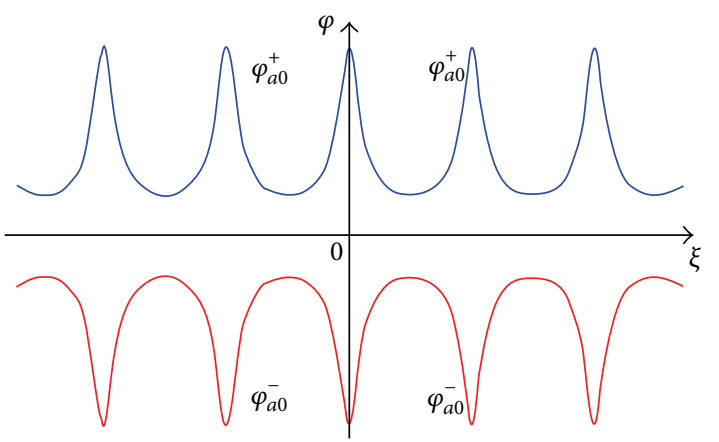

(a)

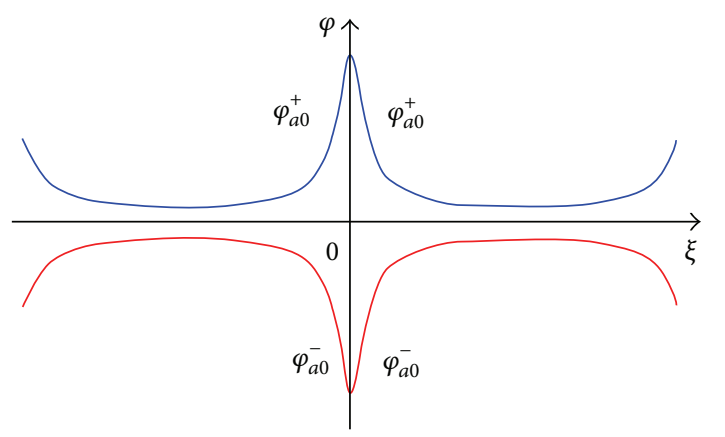

(c)

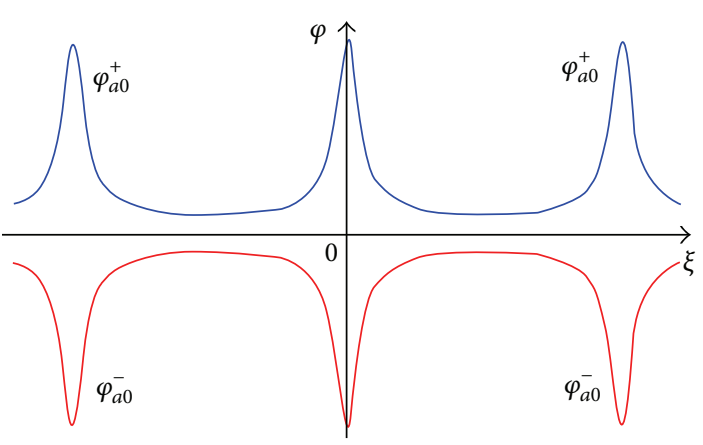

(b)

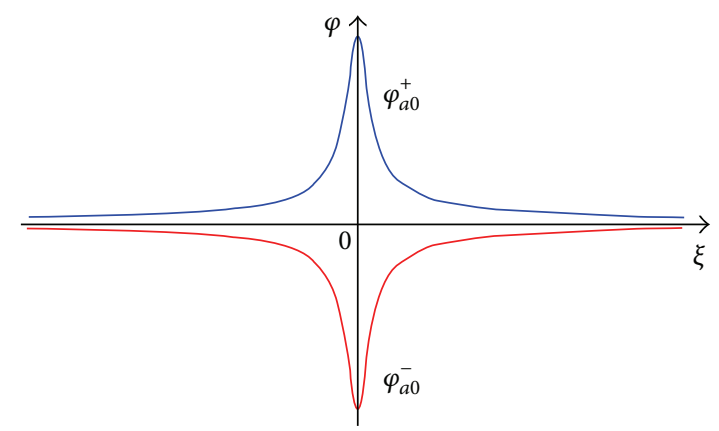

(d)

FIGURE 4: (The symmetric solitary waves are bifurcated from the symmetric periodic waves.) The varying process for the figures of $\varphi_{a 0}^{ \pm}$when $(q, p) \in A_{12}$ and $p \rightarrow 0-0$, where $r=-1, q=4$ and (a) $p=0-0.5$, (b) $p=0-0.1$, (c) $p=0-0.01$, and (d) $p=0-0.001$.

$\left(1^{\circ}\right)$ For the case of $\mu>0$, there are six properties as follows.

$\left(1^{\circ}\right)_{\mathrm{a}}$ If $(q, p) \in l_{2}$, that is, $r>0, q<0$, and $r=$ $3 q^{2} / 16 p$, then $\varphi_{e}^{ \pm}$and $\varphi_{f}^{ \pm}$become

$$
\begin{aligned}
& \varphi_{e 1}^{\mp}=\mp \sqrt{\frac{4 \mu p}{16 p e^{-2 \sqrt{p} \xi}-\mu q}}, \\
& \varphi_{f 1}^{\mp}=\mp \sqrt{\frac{4 \mu p}{16 p e^{2 \sqrt{p} \xi}-\mu q}},
\end{aligned}
$$

which represent four low-kink waves (refer to Figure $9(d))$. Specially, let $\mu=-(16 p / q)>0$, then $\varphi_{e 1}^{\mp}=\varphi_{c 0}^{\mp}$ and $\varphi_{f 1}^{\mp}=\varphi_{b 0}^{\mp}$.

$\left(1^{\circ}\right)_{\mathrm{b}}$ If $(q, p) \in A_{1}, A_{2}, A_{3}$, and $\mu \neq\left|\mu_{0}\right|$, then $\varphi_{e}^{ \pm} \neq \varphi_{f}^{ \pm}$, and they represent four tall-kink waves (refer to Figure $9(a))$. Specially, when $(q, p) \in A_{3}$ and $p \rightarrow 3 q^{2} / 16 r-0$, then $\varphi_{e}^{ \pm}$and $\varphi_{f}^{ \pm}$become $\varphi_{e 1}^{\mp}$ and $\varphi_{f 1}^{\mp}$, and for the varying process, see Figure 9.

$\left(1^{\circ}\right)_{\mathrm{c}}$ If $(q, p) \in A_{4}, A_{11}, A_{12}, l_{6}$, and $\mu \neq\left|\mu_{0}\right|$, then $\varphi_{e}^{ \pm} \neq \varphi_{f}^{ \pm}$. When $(q, p) \in A_{4}$, they represent four antisymmetric solitary waves (refer to Figure $10(a))$. When $(q, p) \in A_{11}, A_{12}$, and $l_{6}$, they represent four symmetric solitary waves (refer to Figure 11(a)). Specially, when $(q, p) \in$ $A_{4}$, if $p \rightarrow 3 q^{2} / 16 r+0$, then $\varphi_{e}^{ \pm}$and $\varphi_{f}^{ \pm}$ become $\varphi_{e 1}^{\mp}$ and $\varphi_{f 1}^{\mp}$, and for the varying process, see Figure 10. When $(q, p) \in A_{11}$ and $p \rightarrow$ $q^{2} / 4 r+0$, then $\varphi_{e}^{ \pm}$and $\varphi_{f}^{ \pm}$tend to two trivial solutions $\varphi= \pm \sqrt{-(q / 2 r)}$, and for the varying process, see Figure 11.

$\left(1^{\mathrm{o}}\right)_{\mathrm{d}}$ If $(q, p) \in A_{3}$ and $\mu=\left|\mu_{0}\right|$, then $\varphi_{e}^{ \pm}=\varphi_{f}^{\mp}=\varphi_{g}^{\mp}$ of forms

$$
\varphi_{g}^{\mp}=\mp \frac{\sqrt{(1 / r)(\Delta-q)(2 \Delta+q)} \sinh ((\theta / 2) \xi)}{\sqrt{-q+4 \Delta+(q+2 \Delta) \cosh (\theta \xi)}},
$$

which represent two tall-kink waves and tend to a trivial wave $\varphi=0$ when $p \rightarrow 3 q^{2} / 16 r-0$.

$\left(1^{\mathrm{o}}\right)_{\mathrm{e}}$ If $(q, p) \in A_{4}$ and $\mu=\left|\mu_{0}\right|$, then $\varphi_{e}^{ \pm}=\varphi_{f}^{ \pm}=\varphi_{h}^{\mp}$ of forms

$$
\varphi_{h}^{\mp}=\mp \frac{\sqrt{(1 / r)(\Delta-q)(2 \Delta+q)} \cosh ((\theta / 2) \xi)}{\sqrt{q-4 \Delta+(q+2 \Delta) \cosh (\theta \xi)}},
$$

which represent two antisymmetric solitary waves, tend to a trivial wave $\varphi=0$ when $p \rightarrow 3 q^{2} / 16 r+0$, and tend to two trivial waves $\varphi= \pm \sqrt{-(q / 2 r)}$ when $p \rightarrow q^{2} / 4 r-0$.

$\left(1^{\circ}\right)_{\mathrm{f}}$ If $(q, p) \in A_{11}, A_{12}, l_{6}$ and $\mu=\left|\mu_{0}\right|$, then $\varphi_{e}^{ \pm}=$ $\varphi_{f}^{ \pm}=\varphi_{h}^{ \pm}$which represent two symmetric solitary waves. Specially, when $(q, p) \in A_{11}$ and $p \rightarrow$ $q^{2} / 4 r-0$, then $\varphi_{h}^{ \pm}$tend to two trivial waves $\varphi=$ $\pm \sqrt{-(q / 2 r)}$. 


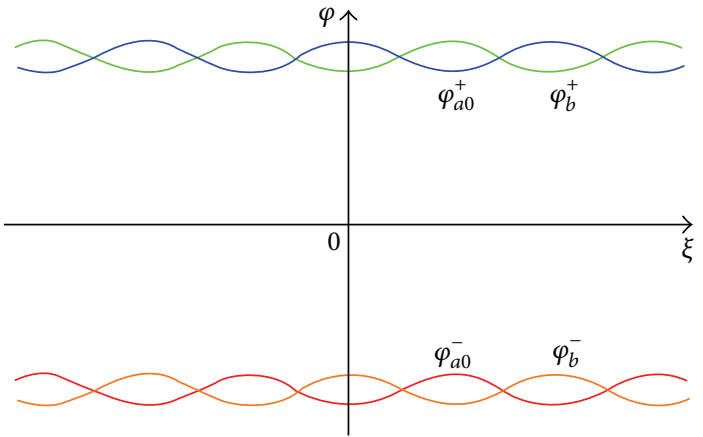

(a)

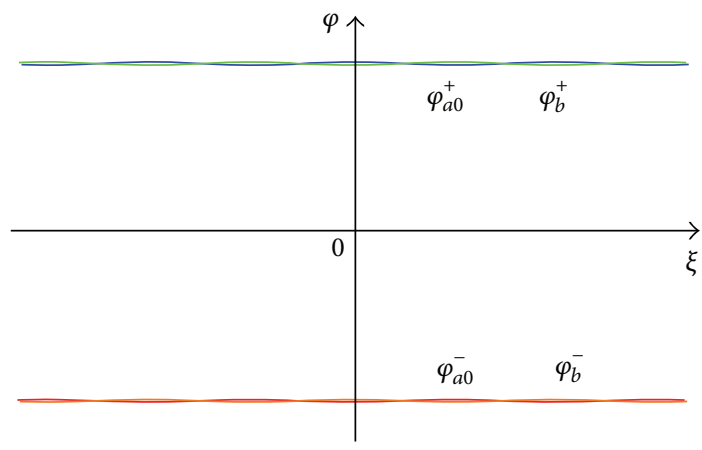

(c)

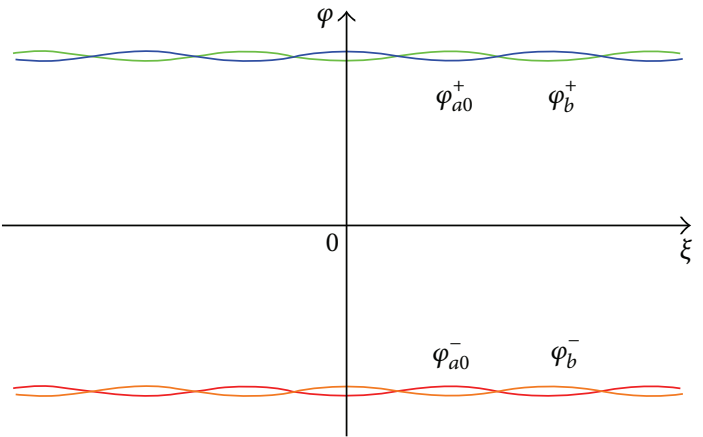

(b)

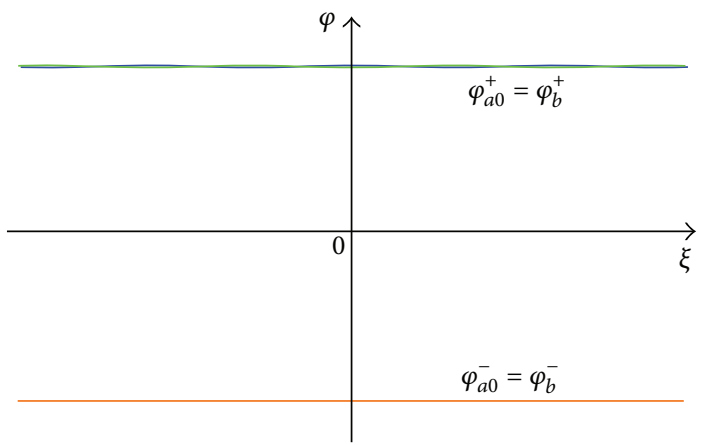

(d)

Figure 5: (The symmetric periodic waves become two trivial waves.) The varying process for the figures of $\varphi_{a 0}^{ \pm}$and $\varphi_{b}^{ \pm}$when $(q, p) \in A_{12}$ and $p \rightarrow 3 q^{2} / 16 r+0$, where $r=-1, q=4$, and $l_{6}: p=3 q^{2} / 16 r=-3$ and (a) $p=-3+10^{-1}$, (b) $p=-3+10^{-2},(\mathrm{c}) p=-3+10^{-3}$, and (d) $p=-3+10^{-6}$.

$\left(2^{\circ}\right)$ For the case of $\mu<0$, there are five properties as follows.

$\left(2^{\circ}\right)_{\mathrm{a}}$ If $(q, p) \in l_{2}$, then $\varphi_{e 1}^{ \pm}$and $\varphi_{f 1}^{ \pm}$represent four 1 blow-up waves (refer to Figure 12(d)). Specially, let $\mu=16 p / q<0$, then $\varphi_{e 1}^{ \pm}=\varphi_{e 0}^{ \pm}$and $\varphi_{f}^{ \pm}=\varphi_{d 2}^{\mp}$.

$\left(2^{\circ}\right)_{\mathrm{b}}$ If $(q, p) \in A_{1}, A_{2}, A_{3}, A_{4}$ and $\mu \neq-\left|\mu_{0}\right|$, then $\varphi_{e}^{ \pm} \neq \varphi_{f}^{ \pm}$, and they represent four pairs of 1-blow-up waves (refer to Figure 12(a)). In particular, when $(q, p) \in A_{4}$, if $p \rightarrow 3 q^{2} / 16 r+0$, then the four pairs of 1-blow-up waves become two pairs of 1-blow-up waves, and the varying process is displayed in Figure 12. If $p \rightarrow q^{2} / 4 r-0$, then the four pairs of 1-blow-up waves become two trivial waves $\varphi= \pm \sqrt{-(q / 2 r)}$.

$\left(2^{\circ}\right)_{\mathrm{c}}$ If $(q, p) \in A_{11}, A_{12}, l_{6}$ and $\mu \neq-\left|\mu_{0}\right|$, then $\varphi_{e}^{ \pm} \neq \varphi_{f}^{ \pm}$, and they represent four tall-kink waves.

$\left(2^{\circ}\right)_{\mathrm{d}}$ If $\mu=-\left|\mu_{0}\right|$, when $(q, p) \in A_{3}$, then $\varphi_{e}^{ \pm}=\varphi_{f}^{ \pm}=$ $\varphi_{h}^{\mp}$ which represent two pairs of 1-blow-up waves. When $(q, p) \in A_{4}$, then $\varphi_{e}^{ \pm}=\varphi_{f}^{\mp}=\varphi_{g}^{\mp}$ which represent two pairs of 1-blow-up waves.

$\left(2^{\circ}\right)_{\mathrm{e}}$ If $(q, p) \in A_{11}, A_{12}, l_{6}$ and $\mu=-\left|\mu_{0}\right|$, then $\varphi_{e}^{ \pm}=$ $\varphi_{f}^{\mp}=\varphi_{g}^{\mp}$ which represent two tall-kink waves.

\subsection{When the Orbit $\Gamma$ Is Defined by $H(\varphi, y)=H\left(\varphi_{2}, 0\right)$}

Proposition 3. (i) If $(q, p)$ belongs to one of $A_{3}, A_{4}, A_{11}$, and $l_{2}$, then (1) have four nonlinear wave solutions

$$
\begin{array}{ll}
u_{i}^{ \pm}=\frac{\beta}{c^{2}-c_{s}^{2}}\left(\varphi_{i}^{ \pm}\right)^{2}, & E_{i}^{ \pm}=\varphi_{i}^{ \pm} e^{i(\gamma x-\omega t)}, \\
u_{j}^{ \pm}=\frac{\beta}{c^{2}-c_{s}^{2}}\left(\varphi_{j}^{ \pm}\right)^{2}, & E_{j}^{ \pm}=\varphi_{j}^{ \pm} e^{i(\gamma x-\omega t)},
\end{array}
$$

where

$$
\begin{gathered}
\varphi_{i}^{ \pm}= \pm \sqrt{\frac{(q+\Delta)(2 \Delta-q)}{r(q+4 \Delta+(q-2 \Delta) \cos (\eta \xi))}} \cos \left(\frac{\eta}{2} \xi\right), \\
\varphi_{j}^{ \pm}= \pm \sqrt{\frac{(q+\Delta)(2 \Delta-q)}{r(q+4 \Delta-(q-2 \Delta) \cos (\eta \xi))}} \sin \left(\frac{\eta}{2} \xi\right), \\
\eta=\sqrt{\frac{1}{r}(4 p r-q(q+\Delta)) .}
\end{gathered}
$$

When $(q, p) \in A_{3}, A_{4}$, or $l_{2}$, then $\varphi_{i}^{ \pm}$and $\varphi_{j}^{ \pm}$represent periodic blow-up wave solutions (refer to Figure 13(a)). When $(q, p) \in A_{11}$, then $\varphi_{i}^{ \pm}$and $\varphi_{j}^{ \pm}$represent periodic wave solutions (refer to Figure 14(a)).

(ii) If $(q, p) \in l_{1}$ or $l_{7}$, then (1) have two fractional wave solutions

$$
u_{k}^{ \pm}=\frac{\beta}{c^{2}-c_{s}^{2}}\left(\varphi_{k}^{ \pm}\right)^{2}, \quad E_{k}^{ \pm}=\varphi_{k}^{ \pm} e^{i(\gamma x-\omega t)},
$$




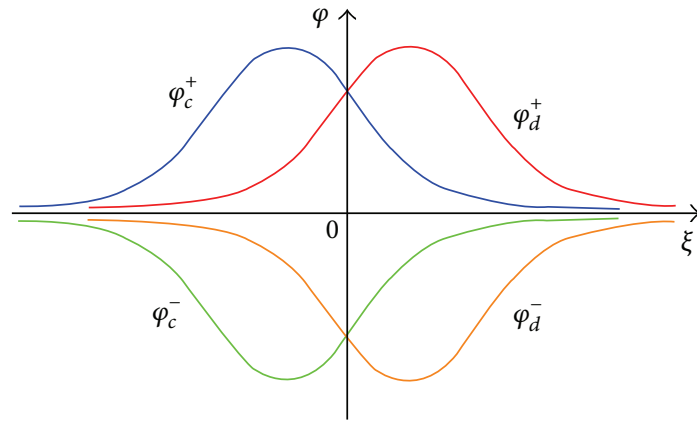

(a)

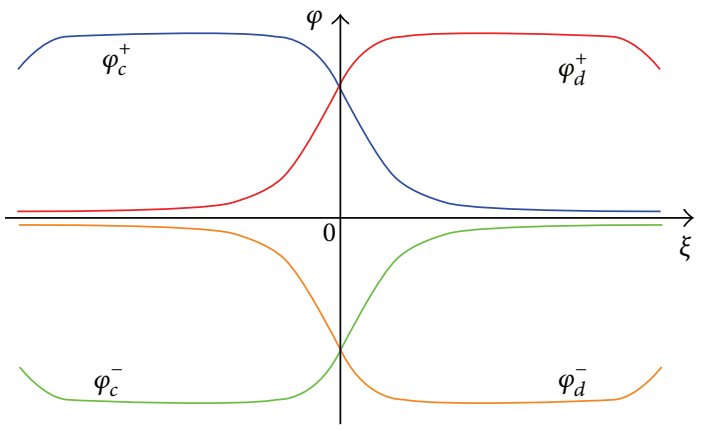

(c)

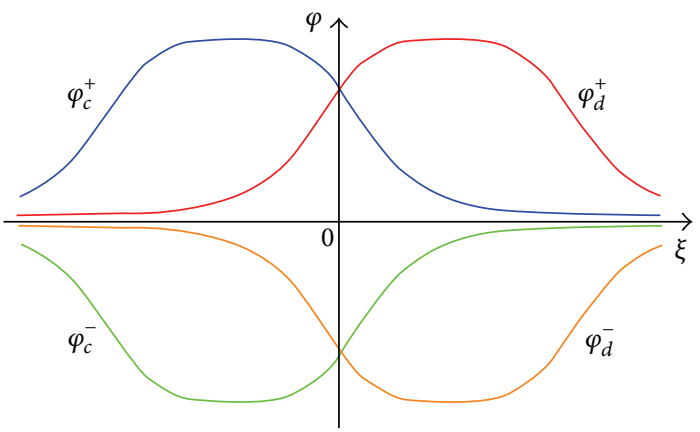

(b)

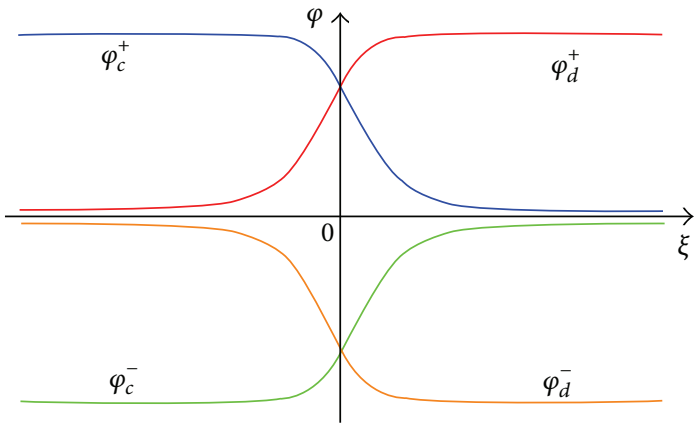

(d)

Figure 6: (The four low-kink waves are bifurcated from four symmetric solitary waves.) The varying process for the figures of $\varphi_{c}^{ \pm}$and $\varphi_{d}^{ \pm}$ when $(q, p) \in A_{3}, \lambda>0, \lambda \neq \sqrt{\lambda_{0} / 12}$ and $p \rightarrow 3 q^{2} / 16 r-0$, where $r=1, q=-4, \lambda=4$, and $l_{2}: p=3 q^{2} / 16 r=3$ and (a) $p=3-10^{-1},(\mathrm{~b})$ $p=3-10^{-3}$, (c) $p=3-10^{-7}$, and (d) $p=3-10^{-9}$.

where

$$
\varphi_{k}^{ \pm}= \pm \sqrt{\frac{-q}{2 r}} \frac{q \xi}{\sqrt{q^{2} \xi^{2}-12 r}} .
$$

When $(q, p) \in l_{1}, \varphi_{k}^{ \pm}$represent two 1-blow-up waves (refer to Figure $13(d))$. When $(q, p) \in l_{7}, \varphi_{k}^{ \pm}$represent two tall-kink waves (refer to Figure 14(d)).

(iii) If $(q, p) \in A_{4}$ and $p \rightarrow q^{2} / 4 r-0$, then the periodic blow-up waves $\varphi_{i}^{ \pm}$tend to two trivial solutions $\varphi=$ $\pm \sqrt{-(q / 2 r)}$, while $\varphi_{j}^{ \pm}$tend to the 1-blow-up waves $\varphi_{k}^{ \pm}$, and the varying process is displayed in Figure 13. If $(q, p) \in A_{11}$ and $p \rightarrow q^{2} / 4 r+0$, then the periodic waves $\varphi_{i}^{ \pm}$tend to two trivial solutions $\varphi= \pm \sqrt{-(q / 2 r)}$, while $\varphi_{j}^{ \pm}$tend to the tall-kink waves $\varphi_{k}^{ \pm}$, and for the varying process, see Figure 14 .

\section{The Derivations of Main Results}

To derive our results, substituting $(23)$ and $u(x, t)=u(\xi)$ with $\xi=x-c t$ into (1), it follows that

$$
\begin{gathered}
\left(c^{2}-c_{s}^{2}\right) u^{\prime \prime}=\beta\left(\varphi^{2}\right)^{\prime \prime} \\
\alpha \varphi^{\prime \prime}+i(2 \alpha \gamma-c) \varphi^{\prime}+\left(w-\alpha \gamma^{2}\right) \varphi \\
-\delta_{1} u \varphi+\delta_{2} \varphi^{3}+\delta_{3} \varphi^{5}=0 .
\end{gathered}
$$

Integrating the first equation of (52) twice with respect to $\xi$ and taking the integral constants to zero, we get (22). Substituting (22) into the second equation of (52) and letting $c=2 \alpha \gamma$, it follows that

$$
\varphi^{\prime \prime}=p \varphi+q \varphi^{3}+r \varphi^{5} .
$$

Form (53) we obtain the planar system

$$
\begin{gathered}
\frac{d \varphi}{d \xi}=y, \\
\frac{d y}{d \xi}=p \varphi+q \varphi^{3}+r \varphi^{5},
\end{gathered}
$$

with the first integral

$$
H(\varphi, y)=y^{2}-\left(p \varphi^{2}+\frac{q}{2} \varphi^{4}+\frac{r}{3} \varphi^{6}\right)=h .
$$

According to the qualitative theory, we obtain the bifurcation phase portraits of system (54) as Figures 15 and 16. Using the information given by Figures 15 and 16, we give derivations to Propositions 1,2 , and 3 , respectively.

3.1. The Derivations to Proposition 1. When the orbit $\Gamma$ is defined by $H(\varphi, y)=H(0,0)$, from (55) we obtain

$$
y= \pm \sqrt{p \varphi^{2}+\frac{q}{2} \varphi^{4}+\frac{r}{3} \varphi^{6}} .
$$




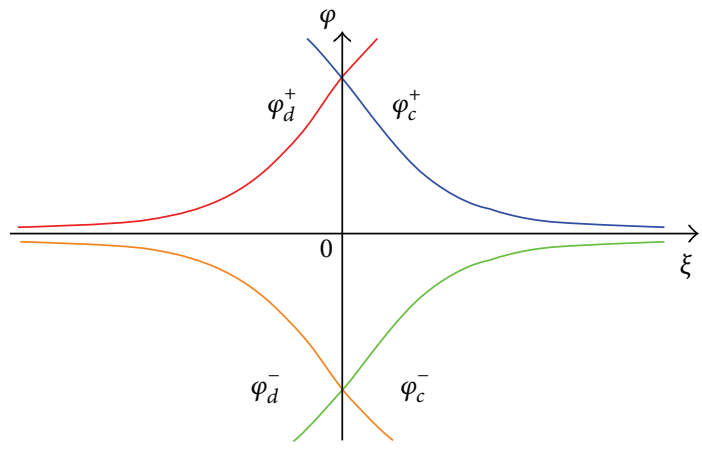

(a)

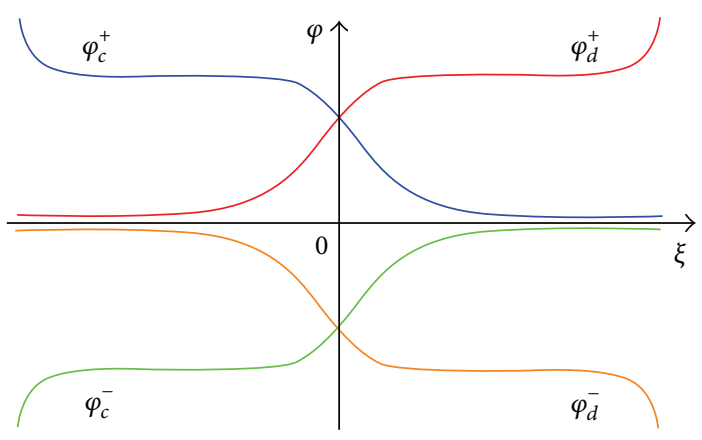

(c)

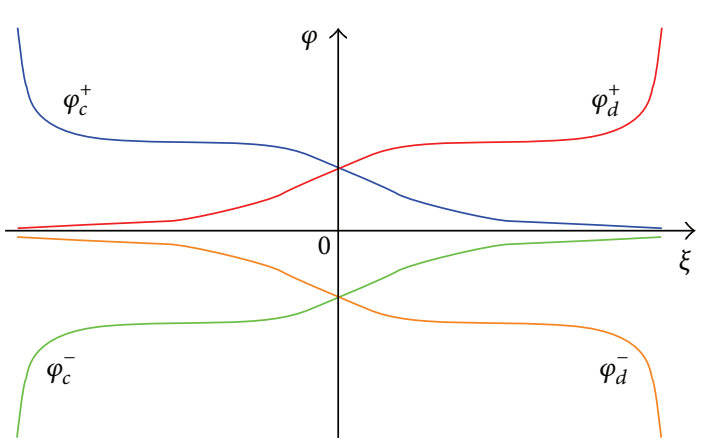

(b)

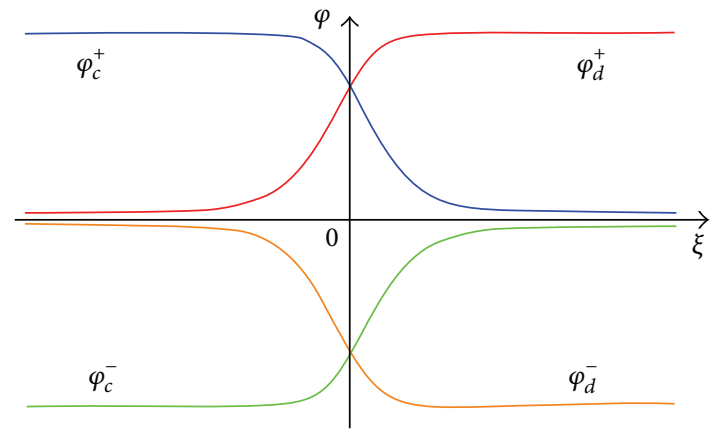

(d)

FIGURE 7: (The four low-kink waves are bifurcated from four 1-blow-up waves.) The varying process for the figures of $\varphi_{c}^{ \pm}$and $\varphi_{d}^{ \pm}$when $(q, p) \in A_{4}, \lambda>0, \lambda \neq \sqrt{\lambda_{0} / 12}$, and $p \rightarrow 3 q^{2} / 16 r+0$, where $r=1, q=-4, \lambda=4$, and $l_{2}: p=3 q^{2} / 16 r=3$ and (a) $p=3+10^{-1},(\mathrm{~b})$ $p=3+10^{-3}$, (c) $p=3+10^{-6}$, and (d) $p=3+10^{-9}$.

Substituting (56) into the first equation of (54) and integrating it, we have

$$
\int_{l}^{\varphi} \frac{d s}{\sqrt{p s^{2}+(q / 2) s^{4}+(r / 3) s^{6}}}=\xi
$$

where $l$ is an arbitrary constant or $\pm \infty$.

For $p=0$, letting $l=\sqrt{-(3 q / 2 r)}$ or $\pm \infty$ and completing the above integral, it follows that

$$
\varphi= \pm \sqrt{\frac{6 q}{3 q^{2} \xi^{2}-4 r}}
$$

which yields $\varphi_{a}^{ \pm}$as (29). that

For $p<0$, completing (57) and solving for $\varphi$, it follows

$$
\varphi= \pm \sqrt{\frac{-12 p}{3 q-\sqrt{9 q^{2}-48 p r} \sin (\lambda+2 \sqrt{-p} \xi)}},
$$

where $\lambda=\lambda(l)$ is an arbitrary real constant. Let $\lambda= \pm(\pi / 2)$, respectively, and we obtain the solutions $\varphi_{a 0}^{ \pm}$and $\varphi_{b}^{ \pm}$as (3) and (31).

When $(q, p) \in A_{1}$, that is, $r>0, q>0$, and $p<0$, let

$$
\begin{aligned}
& f(p)=3 q-\sqrt{9 q^{2}-48 p r} \cos (2 \sqrt{-p} \xi), \\
& g(p)=3 q+\sqrt{9 q^{2}-48 p r} \cos (2 \sqrt{-p} \xi) .
\end{aligned}
$$

Thus, we have

$$
\begin{aligned}
\lim _{p \rightarrow 0-0} \varphi_{a 0}^{ \pm} & =\lim _{p \rightarrow 0-0} \pm \sqrt{\frac{-12 p}{f(p)}} \\
& = \pm \sqrt{\lim _{p \rightarrow 0-0} \frac{-12}{f^{\prime}(p)}} \\
& = \pm\left(\operatorname { l i m } _ { p \rightarrow 0 - 0 } \left(2 \sqrt{9 q^{2}-48 p r}\right.\right. \\
& \times\left(\left(3 q^{2}-16 p r\right) \xi^{2}\right. \\
& \times(\sin (2 \sqrt{-p} \xi) / 2 \sqrt{-p} \xi) \\
& \left.\left.\left.= \pm \sqrt{\frac{6 q}{3 q^{2} \xi^{2}-4 r}},-4 r \cos (2 \sqrt{-p} \xi)\right)^{-1}\right)\right)^{1 / 2} \\
& =\varphi_{a}^{ \pm}
\end{aligned}
$$$$
\lim _{p \rightarrow 0-0} \varphi_{b}^{ \pm}=\lim _{p \rightarrow 0-0} \pm \sqrt{\frac{-12 p}{g(p)}}
$$$$
= \pm \sqrt{\lim _{p \rightarrow 0-0} \frac{-12 p}{g(p)}}
$$$$
=0 \text {. }
$$ 


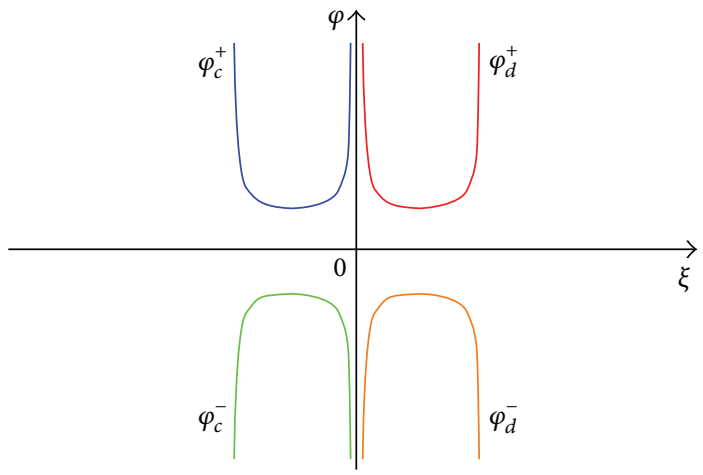

(a)

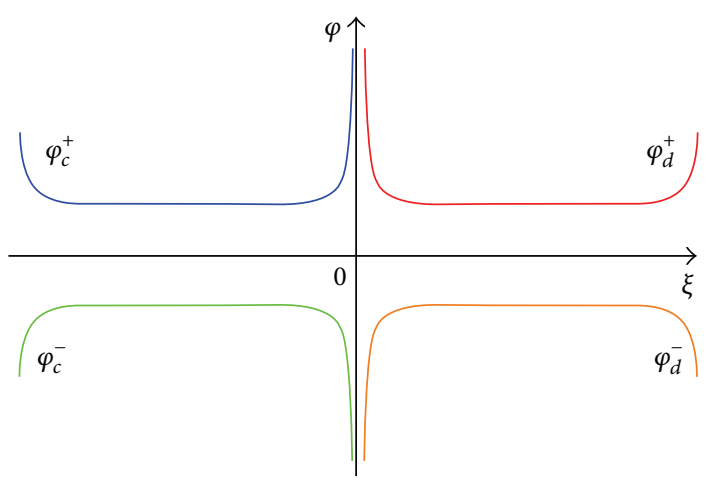

(c)

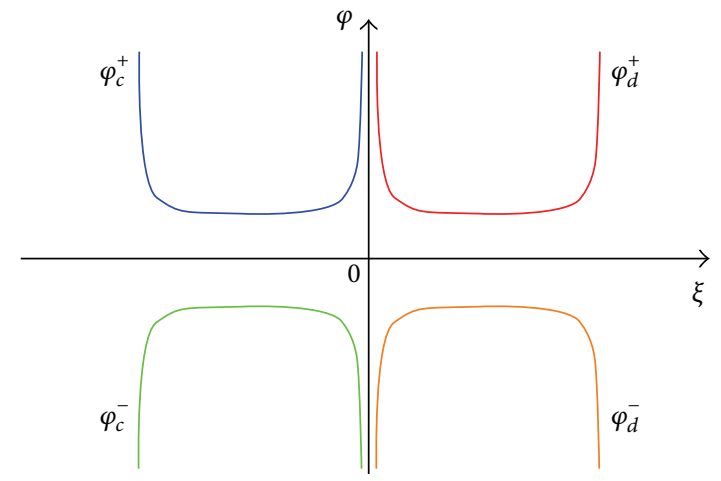

(b)

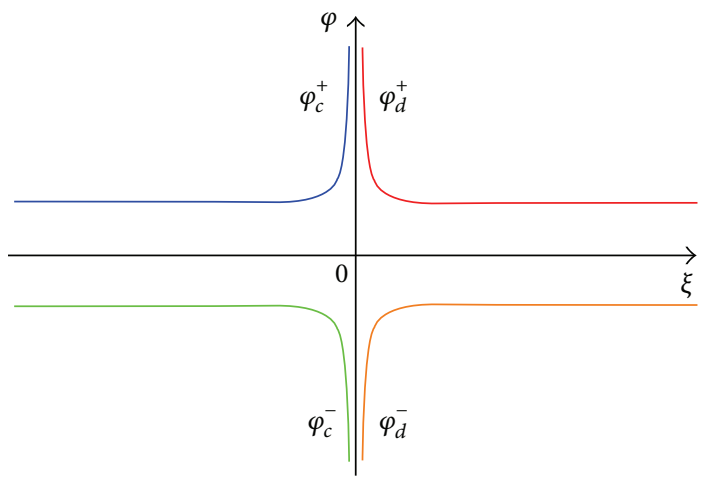

(d)

Figure 8: (The four 2-blow-up waves become four 1-blow-up waves.) The varying process for the figures of $\varphi_{c}^{ \pm}$and $\varphi_{d}^{ \pm}$when $(q, p) \in A_{3}, \lambda<$ $0, \lambda \neq-\sqrt{\lambda_{0} / 12}$, and $p \rightarrow 3 q^{2} / 16 r-0$, where $r=1, q=-4, \lambda=-4$, and $l_{2}: p=3 q^{2} / 16 r=3$ and (a) $p=3-10^{-1}$, (b) $p=3-10^{-3}$, (c) $p=3-10^{-6}$, and (d) $p=3-10^{-9}$.

When $(q, p) \in A_{2}$, that is, $r>0, q<0$, and $p<0$, similarly we get

$$
\lim _{p \rightarrow 0-0} \varphi_{b}^{ \pm}=\varphi_{a}^{ \pm}
$$

When $(q, p) \in A_{12}$, that is, $r<0, q>0$, and $p<0$, if $p \rightarrow 3 q^{2} / 16 r+0$, then $f(p)$ and $g(p) \rightarrow 0$.

Thus,

$$
\lim _{p \rightarrow 0-0} \varphi_{a 0}^{ \pm}\left(\text {or } \varphi_{b}^{ \pm}\right)= \pm \sqrt{-\frac{3 q}{4 r}} .
$$
that

For $p>0$, completing (57) and solving for $\varphi$, it follows

$$
\varphi= \pm \sqrt{\frac{4 \lambda p e^{2 \sqrt{p} \xi}}{\lambda^{2} e^{4 \sqrt{p} \xi}+\left(q^{2} / 4-4 p r / 3\right)-\lambda q e^{2 \sqrt{p} \xi}}}
$$

where $\lambda=\lambda(l)$ is an arbitrary real constant.

Note that if $\varphi(\xi)$ is a solution of (53), so is $\varphi(-\xi)$. Thus, from (64) we obtain the solutions $\varphi_{c}^{ \pm}$and $\varphi_{d}^{ \pm}$as in (33).
For the case of $\lambda>0$. When $(q, p) \in l_{2}$, that is, $r>0, q<$ 0 and $r=3 q^{2} / 16 p$. In (33) letting $r=3 q^{2} / 16 p$, we get (34). Furthermore, in (34) letting $\lambda=-q>0$, it follows that

$$
\begin{aligned}
\varphi_{c 1}^{ \pm} & = \pm \sqrt{\frac{4 p}{-q\left(e^{2 \sqrt{p} \xi}+1\right)}} \\
& = \pm \sqrt{\frac{4 p e^{-\sqrt{p} \xi}}{-q\left(e^{\sqrt{p} \xi}+e^{-\sqrt{p} \xi}\right)}} \\
& = \pm \sqrt{\frac{2 p}{-q}\left(1-\frac{e^{\sqrt{p} \xi}-e^{-\sqrt{p} \xi}}{e^{\sqrt{p} \xi}+e^{-\sqrt{p} \xi}}\right)} \\
& = \pm \sqrt{\frac{2 p}{-q}(1-\tanh (\sqrt{p} \xi))} \\
& = \pm \sqrt{\frac{2 p}{q}(-1+\tanh (\sqrt{p} \xi))} \\
& =\varphi_{b 0}^{ \pm}(\operatorname{see}(4))
\end{aligned}
$$




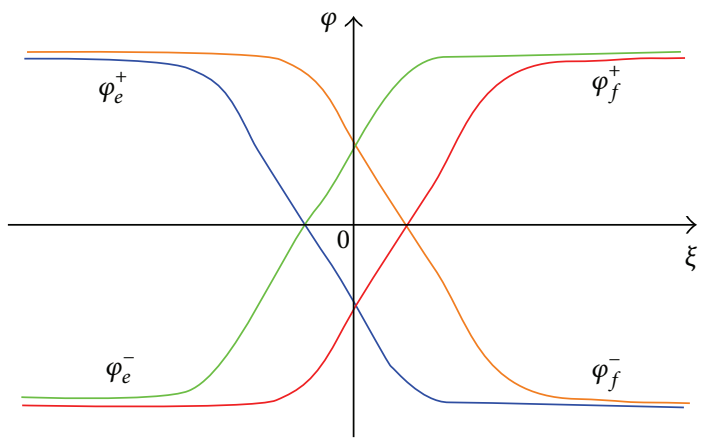

(a)

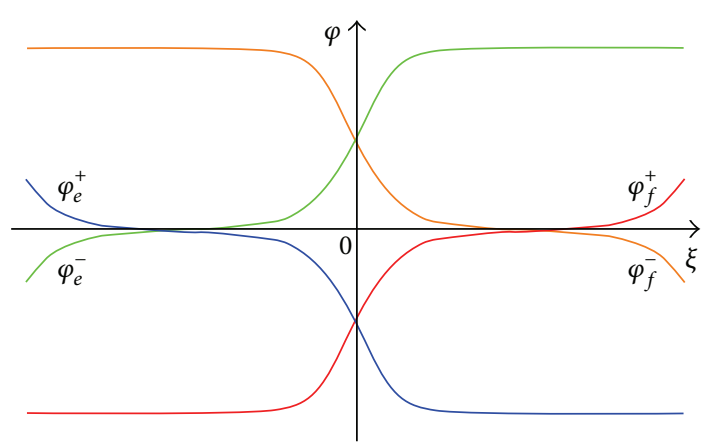

(c)

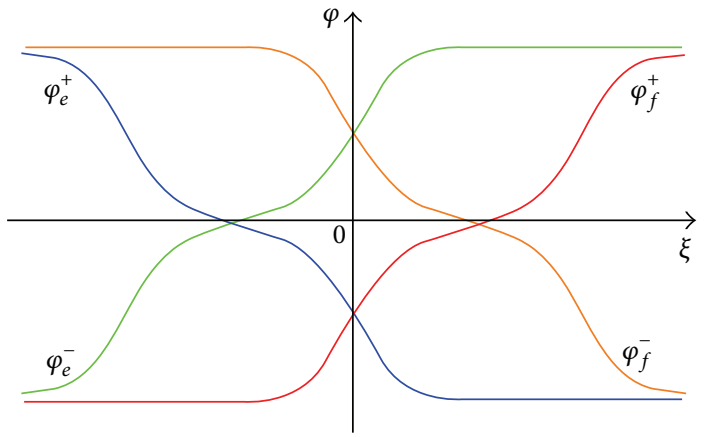

(b)

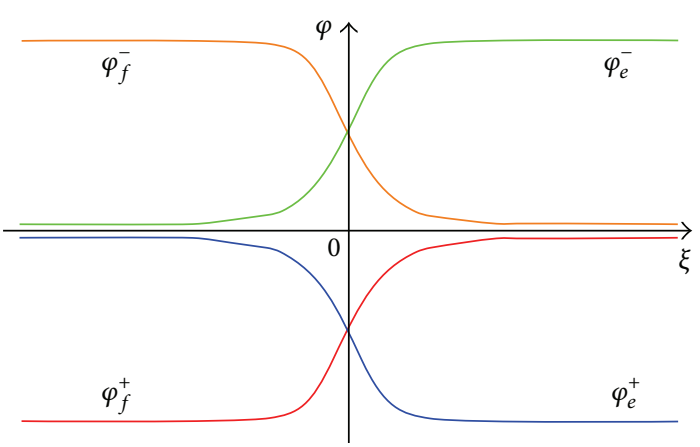

(d)

FIGURE 9: (The four low-kink waves are bifurcated from the four tall-kink waves.) The varying process for the figures of $\varphi_{e}^{ \pm}$and $\varphi_{f}^{ \pm}$when $\mu>0, \mu \neq\left|\mu_{0}\right|,(q, p) \in A_{3}$, and $p \rightarrow 3 q^{2} / 16 r-0$, where $r=1, q=-4, l_{2}: p=3 q^{2} / 16 r=3$, and $\mu=4$ and (a) $p=3-0.5$, (b) $p=3-10^{-2}$, (c) $p=3-10^{-4}$, and (d) $p=3-10^{-6}$.

$$
\begin{aligned}
\varphi_{d 1}^{ \pm} & = \pm \sqrt{\frac{4 p}{-q\left(e^{-2 \sqrt{p} \xi}+1\right)}} \\
& = \pm \sqrt{\frac{4 p e^{\sqrt{p} \xi}}{-q\left(e^{\sqrt{p} \xi}+e^{-\sqrt{p} \xi}\right)}} \\
& = \pm \sqrt{\frac{2 p}{-q}\left(1+\frac{e^{\sqrt{p} \xi}-e^{-\sqrt{p} \xi}}{e^{\sqrt{p} \xi}+e^{-\sqrt{p} \xi}}\right)} \\
& = \pm \sqrt{\frac{2 p}{-q}(1+\tanh (\sqrt{p} \xi))} \\
& = \pm \sqrt{\frac{2 p}{q}(-1-\tanh (\sqrt{p} \xi))}=\varphi_{c 0}^{ \pm} \quad(\operatorname{see}(5)) .
\end{aligned}
$$

When $\lambda=\sqrt{\lambda_{0} / 12}$, by (33) it follows that

$$
\begin{aligned}
\varphi_{c}^{ \pm}=\varphi_{d}^{ \pm} & = \pm \sqrt{\frac{24 p}{-6 q+\sqrt{9 q^{2}-48 p r}\left(e^{\sqrt{p} \xi}+e^{-\sqrt{p} \xi}\right)}} \\
& = \pm \sqrt{\frac{12 p}{-3 q+\sqrt{9 q^{2}-48 p r} \cosh (2 \sqrt{p} \xi)}} \\
& =\varphi_{d 0}^{ \pm}(\operatorname{see}(6)) .
\end{aligned}
$$

For the case of $\lambda<0$, similarly we can obtain (7), (35), and (36), and here we omit the process. Hereto, we have completed the derivations for Proposition 1.

3.2. The Derivations to Proposition 2. When the orbit $\Gamma$ is defined by $H(\varphi, y)=H\left(\varphi_{1}, 0\right)$, from (55) we obtain

$$
\begin{aligned}
y & = \pm \sqrt{p \varphi^{2}+\frac{q}{2} \varphi^{4}+\frac{r}{3} \varphi^{6}+H\left(\varphi_{1}, 0\right)} \\
& = \pm \sqrt{\frac{r}{3}\left(\varphi_{1}^{2}-\varphi^{2}\right)^{2}\left(\varphi^{2}+\mu_{0}\right)}
\end{aligned}
$$

where $\varphi_{1}$ and $\mu_{0}$ are given in (25) and (40). Substituting (67) into the first equation of (54) and integrating it, we have

$$
\int_{m}^{\varphi} \frac{d s}{\sqrt{(r / 3)\left(\varphi_{1}^{2}-s^{2}\right)^{2}\left(s^{2}+\mu_{0}\right)}}=\xi
$$

where $m$ is an arbitrary constant or $\pm \infty$.

Completing the above integral and solving for $\varphi$, it follows that

$$
\varphi= \pm \sqrt{\varphi_{1}^{2}-\frac{4 a_{1} \mu e^{\theta \xi}}{\mu^{2} e^{2 \theta \xi}-2 \mu b_{1} e^{\theta \xi}+b_{1}^{2}-4 a_{1}}}
$$




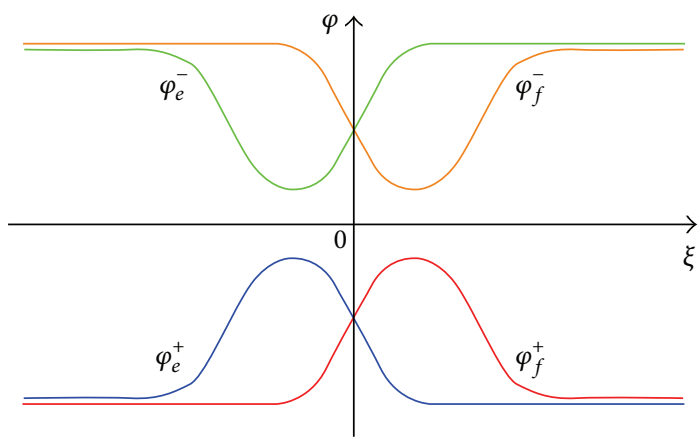

(a)

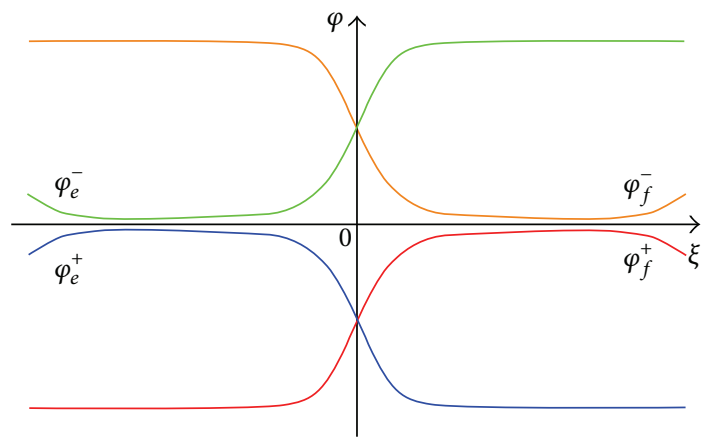

(c)

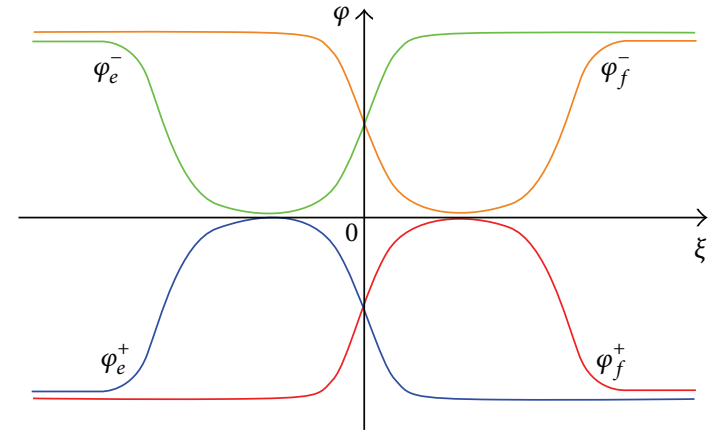

(b)

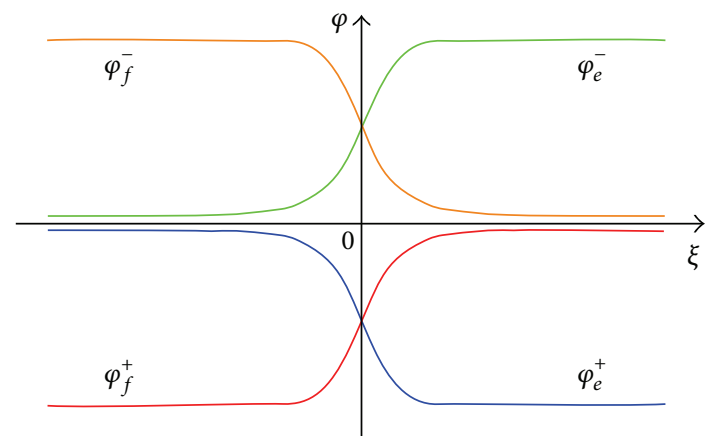

(d)

Figure 10: (The four low-kink waves are bifurcated from the four antisymmetric solitary waves.) The varying process for the figures of $\varphi_{e}^{ \pm}$ and $\varphi_{f}^{ \pm}$when $\mu>0, \mu \neq\left|\mu_{0}\right|, \quad(q, p) \in A_{4}$, and $p \rightarrow 3 q^{2} / 16 r+0$, where $r=1, q=-4, l_{2}: p=3 q^{2} / 16 r=3$, and $\mu=4$ and (a) $p=3+10^{-1}$, (b) $p=3+10^{-3}$, (c) $p=3+10^{-5}$, and (d) $p=3+10^{-7}$.

where $\theta$ is given in (39), $\mu=\mu(m)$ is an arbitrary constant, and

$$
a_{1}=\frac{3 \Delta(\Delta-q)}{4 r^{2}}, \quad b_{1}=\frac{q-4 \Delta}{2 r}
$$

Similar to the derivations for $\varphi_{c}^{ \pm}$and $\varphi_{d}^{ \pm}$, we get $\varphi_{e}^{ \pm}$and $\varphi_{f}^{ \pm}$ (see (38)) from (69).

For the case of $\mu>0$, when $(q, p) \in l_{2}$, that is, $r>0, q<$ 0 , and $r=3 q^{2} / 16 p$, then $\theta=2 \sqrt{p}$ and $q+2 \Delta=0$. From (38) and (41), it is easy to check that $\varphi_{e}^{ \pm}$and $\varphi_{f}^{ \pm}$become $\varphi_{e 1}^{\mp}$ and $\varphi_{f 1}^{\mp}$ (see (42)), respectively. Furthermore, in (42) letting $\mu=-(16 p / q)$, it follows that

$$
\begin{aligned}
\varphi_{e 1}^{\mp} & =\mp \sqrt{\frac{-4 p}{q\left(1+e^{-2 \sqrt{p} \xi}\right)}} \\
& =\mp \sqrt{\frac{2 p}{q}(-1-\tanh (\sqrt{p} \xi))} \\
& =\varphi_{c 0}^{\mp}(\operatorname{see}(5)), \\
\varphi_{f 1}^{\mp} & =\mp \sqrt{\frac{-4 p}{q\left(1+e^{2 \sqrt{p} \xi}\right)}}
\end{aligned}
$$

$$
\begin{aligned}
& =\mp \sqrt{\frac{2 p}{q}(-1+\tanh (\sqrt{p} \xi))} \\
& =\varphi_{b 0}^{\mp}(\operatorname{see}(4)) .
\end{aligned}
$$

If $(q, p) \in A_{3}$ and $\mu=\left|\mu_{0}\right|$, that is, $r>0, \mu=\mu_{0}>0$, and $2 \Delta+q>0$, we have

$$
\begin{aligned}
& \varphi_{e}^{ \pm} \\
& = \pm \frac{\sqrt{(\Delta-q) / 2 r}\left(2 \Delta+q-2 \mu r e^{\theta \xi}\right)}{\sqrt{(2 \Delta+q)^{2}+4 \mu r(4 \Delta-q) e^{\theta \xi}+4 \mu^{2} r^{2} e^{2 \theta \xi}}} \\
& = \pm \frac{\sqrt{(\Delta-q) / 2 r}(2 \Delta+q)\left(1-e^{\theta \xi}\right)}{\sqrt{(q+2 \Delta)^{2}+2(q+2 \Delta)(4 \Delta-q) e^{\theta \xi}+(q+2 \Delta)^{2} e^{2 \theta \xi}}} \\
& = \pm \frac{\sqrt{(1 / 2 r)(\Delta-q)(2 \Delta+q)}\left(1-e^{\theta \xi}\right)}{\sqrt{q+2 \Delta+2(4 \Delta-q) e^{\theta \xi}+(q+2 \Delta) e^{2 \theta \xi}}} \\
& = \pm \frac{\sqrt{(1 / 2 r)(\Delta-q)(2 \Delta+q)\left(e^{-\theta \xi / 2}-e^{\theta \xi / 2}\right)}}{\sqrt{2(4 \Delta-q)+(q+2 \Delta)\left(e^{-\theta \xi}+e^{\theta \xi}\right)}}
\end{aligned}
$$




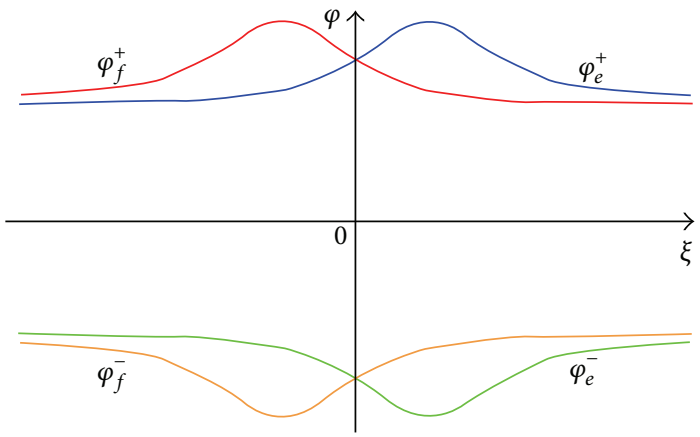

(a)

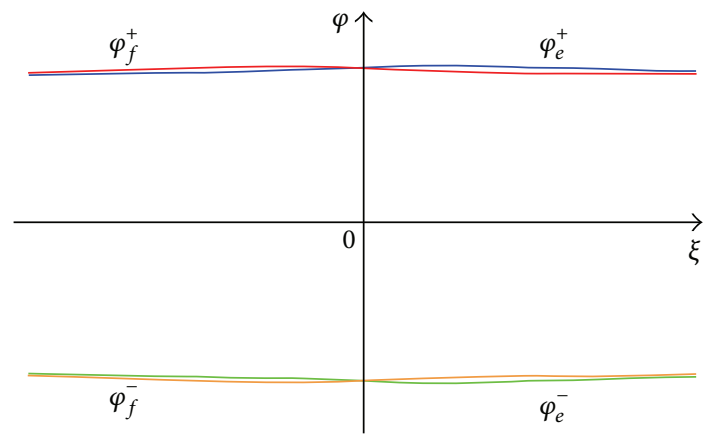

(c)

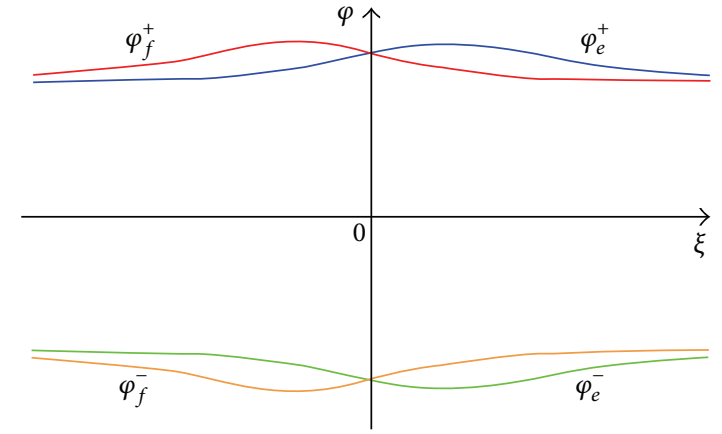

(b)

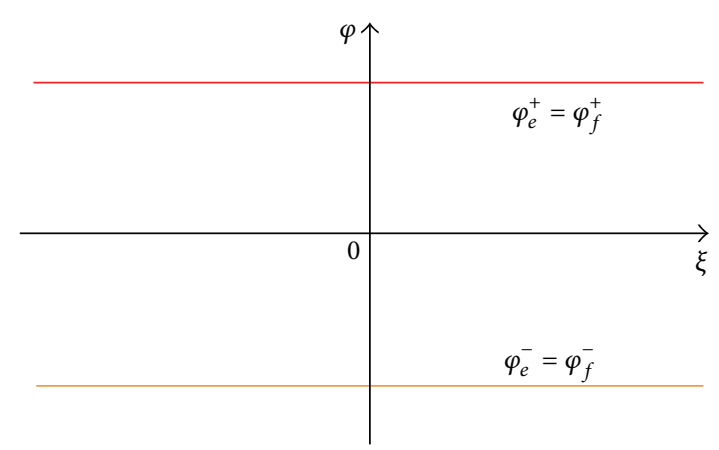

(d)

FIGURE 11: (The four symmetric solitary waves become two trivial waves.) The varying process for the figures of $\varphi_{e}^{ \pm}$and $\varphi_{f}^{ \pm}$when $\mu>$ $0, \mu \neq\left|\mu_{0}\right|,(q, p) \in A_{11}$, and $p \rightarrow q^{2} / 4 r+0$, where $r=-1, q=4, l_{7}: p=q^{2} / 4 r=-4$, and $\mu=1$ and (a) $p=-3.5$, (b) $p=-4+10^{-1},(\mathrm{c})$ $p=-4+10^{-2}$, and (d) $p=-4+10^{-5}$.

$$
\begin{aligned}
& =\mp \frac{\sqrt{(1 / r)(\Delta-q)(2 \Delta+q)} \sinh ((\theta / 2) \xi)}{\sqrt{-q+4 \Delta+(q+2 \Delta) \cosh (\theta \xi)}} \\
& =\varphi_{g}^{\mp}(\operatorname{see}(44)) \text {, } \\
& \varphi_{f}^{ \pm} \\
& = \pm \frac{\sqrt{(\Delta-q) / 2 r}\left(2 \Delta+q-2 \mu r e^{-\theta \xi}\right)}{\sqrt{(2 \Delta+q)^{2}+4 \mu r(4 \Delta-q) e^{-\theta \xi}+4 \mu^{2} r^{2} e^{-2 \theta \xi}}} \\
& = \pm \frac{\sqrt{(\Delta-q) / 2 r}(2 \Delta+q)\left(1-e^{-\theta \xi}\right)}{\sqrt{(q+2 \Delta)^{2}+2(q+2 \Delta)(4 \Delta-q) e^{-\theta \xi}+(q+2 \Delta)^{2} e^{-2 \theta \xi}}} \\
& = \pm \frac{\sqrt{(1 / 2 r)(\Delta-q)(2 \Delta+q)}\left(1-e^{-\theta \xi}\right)}{\sqrt{q+2 \Delta+2(4 \Delta-q) e^{-\theta \xi}+(q+2 \Delta) e^{-2 \theta \xi}}} \\
& = \pm \frac{\sqrt{(1 / 2 r)(\Delta-q)(2 \Delta+q)}\left(e^{\theta \xi / 2}-e^{-\theta \xi / 2}\right)}{\sqrt{2(4 \Delta-q)+(q+2 \Delta)\left(e^{-\theta \xi}+e^{\theta \xi}\right)}} \\
& = \pm \frac{\sqrt{(1 / r)(\Delta-q)(2 \Delta+q)} \sinh ((\theta / 2) \xi)}{\sqrt{-q+4 \Delta+(q+2 \Delta) \cosh (\theta \xi)}} \\
& =\varphi_{g}^{ \pm}(\operatorname{see}(44)) \text {. }
\end{aligned}
$$

If $(q, p) \in A_{4}$ and $\mu=\left|\mu_{0}\right|$, that is, $r>0, \mu=-\mu_{0}>0$, and $2 \Delta+q<0$, we have

$\varphi_{e}^{ \pm}$

$$
\begin{aligned}
& = \pm \frac{\sqrt{(\Delta-q) / 2 r}\left(2 \Delta+q-2 \mu r e^{\theta \xi}\right)}{\sqrt{(2 \Delta+q)^{2}+4 \mu r(4 \Delta-q) e^{\theta \xi}+4 \mu^{2} r^{2} e^{2 \theta \xi}}} \\
& = \pm \frac{\sqrt{(\Delta-q) / 2 r}(2 \Delta+q)\left(1+e^{\theta \xi}\right)}{\sqrt{(q+2 \Delta)^{2}+2(q+2 \Delta)(4 \Delta-q) e^{\theta \xi}+(q+2 \Delta)^{2} e^{2 \theta \xi}}} \\
& =\mp \frac{\sqrt{(1 / 2 r)(\Delta-q)(2 \Delta+q)}\left(1+e^{\theta \xi}\right)}{\sqrt{q+2 \Delta+2(4 \Delta-q) e^{\theta \xi}+(q+2 \Delta) e^{2 \theta \xi}}} \\
& =\mp \frac{\sqrt{(1 / 2 r)(\Delta-q)(2 \Delta+q)\left(e^{-\theta \xi / 2}+e^{\theta \xi / 2}\right)}}{\sqrt{2(4 \Delta-q)+(q+2 \Delta)\left(e^{-\theta \xi}+e^{\theta \xi}\right)}} \\
& =\mp \frac{\sqrt{(1 / r)(\Delta-q)(2 \Delta+q)} \cosh ((\theta / 2) \xi)}{\sqrt{-q+4 \Delta+(q+2 \Delta) \cosh (\theta \xi)}} \\
& =\varphi_{h}^{\mp}(\operatorname{see}(45)),
\end{aligned}
$$




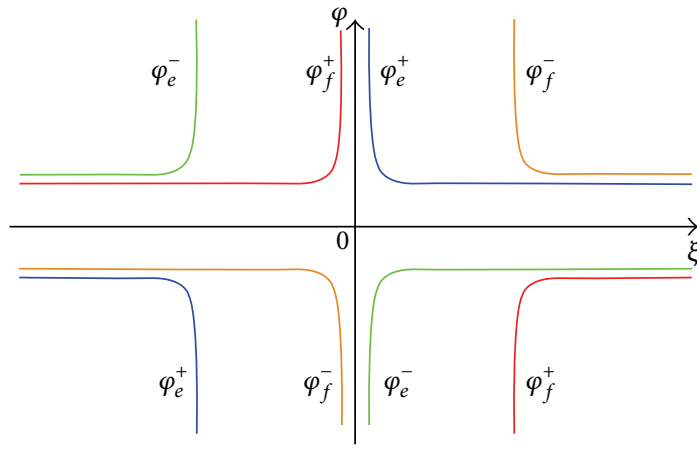

(a)

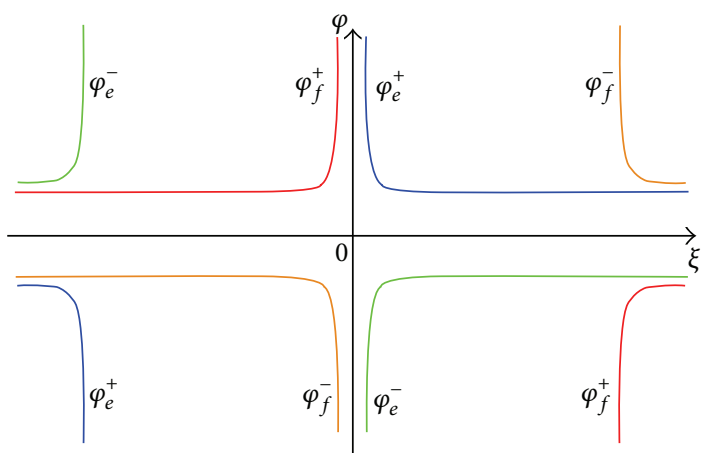

(c)

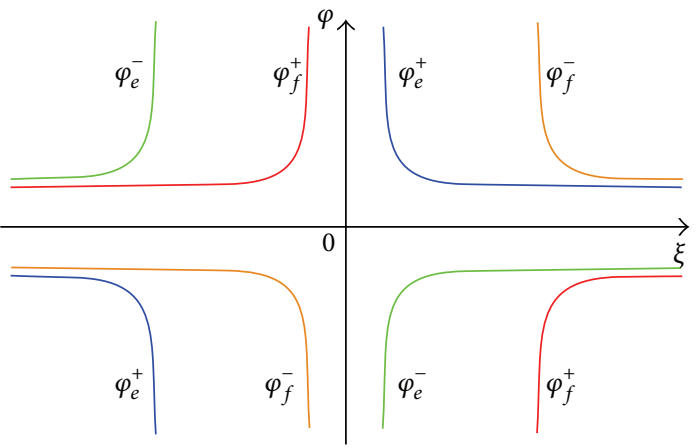

(b)

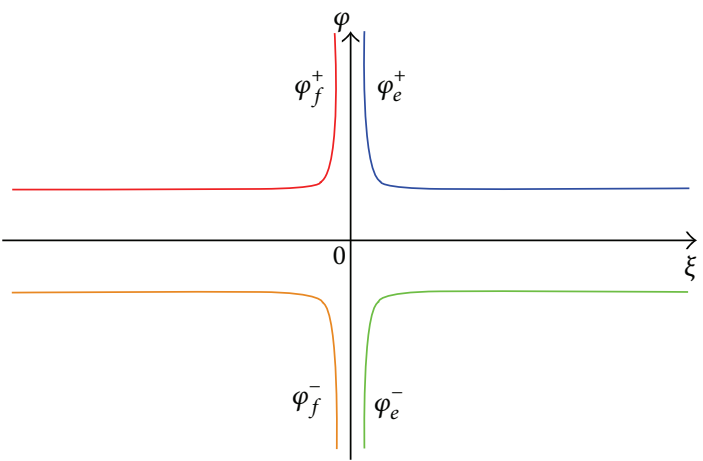

(d)

Figure 12: (The two pairs of 1-blow-up waves are bifurcated from the four pairs of 1-blow-up waves.) The varying process for the figures of $\varphi_{e}^{ \pm}$and $\varphi_{f}^{ \pm}$when $\mu<0, \mu \neq-\left|\mu_{0}\right|,(q, p) \in A_{4}$, and $p \rightarrow 3 q^{2} / 16 r+0$, where $r=1, q=-4, l_{2}: p=3 q^{2} / 16 r=3$, and $\mu=-4$ and (a) $p=3+10^{-2}$, (b) $p=3+10^{-4}$, (c) $p=3+10^{-6}$, and (d) $p=3+10^{-9}$.

$\varphi_{f}^{ \pm}$

$$
\begin{aligned}
& = \pm \frac{\sqrt{(\Delta-q) / 2 r}\left(2 \Delta+q-2 \mu r e^{-\theta \xi}\right)}{\sqrt{(2 \Delta+q)^{2}+4 \mu r(4 \Delta-q) e^{-\theta \xi}+4 \mu^{2} r^{2} e^{-2 \theta \xi}}} \\
& = \pm \frac{\sqrt{(\Delta-q) / 2 r}(2 \Delta+q)\left(1+e^{-\theta \xi}\right)}{\sqrt{(q+2 \Delta)^{2}+2(q+2 \Delta)(4 \Delta-q) e^{-\theta \xi}+(q+2 \Delta)^{2} e^{-2 \theta \xi}}} \\
& =\mp \frac{\sqrt{(1 / 2 r)(\Delta-q)(2 \Delta+q)}\left(1+e^{-\theta \xi}\right)}{\sqrt{q+2 \Delta+2(4 \Delta-q) e^{-\theta \xi}+(q+2 \Delta) e^{-2 \theta \xi}}} \\
& =\mp \frac{\sqrt{(1 / 2 r)(\Delta-q)(2 \Delta+q)\left(e^{-\theta \xi / 2}+e^{\theta \xi / 2}\right)}}{\sqrt{2(4 \Delta-q)+(q+2 \Delta)\left(e^{-\theta \xi}+e^{\theta \xi}\right)}} \\
& =\mp \frac{\sqrt{(1 / r)(\Delta-q)(2 \Delta+q)} \cosh ((\theta / 2) \xi)}{\sqrt{-q+4 \Delta+(q+2 \Delta) \cosh (\theta \xi)}} \\
& =\varphi_{h}^{\mp}(\operatorname{see}(45)) .
\end{aligned}
$$

If $(q, p) \in A_{11}, A_{12}, l_{6}$ and $\mu=\left|\mu_{0}\right|$, that is, $r<0, \mu=$ $-\mu_{0}>0$, and $2 \Delta+q>0$, we have

$\varphi_{e}^{ \pm}$

$= \pm \frac{\sqrt{(\Delta-q) / 2 r}\left(2 \Delta+q-2 \mu r e^{\theta \xi}\right)}{\sqrt{(2 \Delta+q)^{2}+4 \mu r(4 \Delta-q) e^{\theta \xi}+4 \mu^{2} r^{2} e^{2 \theta \xi}}}$

$= \pm \frac{\sqrt{(\Delta-q) / 2 r}(2 \Delta+q)\left(1+e^{\theta \xi}\right)}{\sqrt{(q+2 \Delta)^{2}+2(q+2 \Delta)(4 \Delta-q) e^{\theta \xi}+(q+2 \Delta)^{2} e^{2 \theta \xi}}}$

$= \pm \frac{\sqrt{(1 / 2 r)(\Delta-q)(2 \Delta+q)}\left(1+e^{\theta \xi}\right)}{\sqrt{q+2 \Delta+2(4 \Delta-q) e^{\theta \xi}+(q+2 \Delta) e^{2 \theta \xi}}}$

$= \pm \frac{\sqrt{(1 / 2 r)(\Delta-q)(2 \Delta+q)}\left(e^{-\theta \xi / 2}+e^{\theta \xi / 2}\right)}{\sqrt{2(4 \Delta-q)+(q+2 \Delta)\left(e^{-\theta \xi}+e^{\theta \xi}\right)}}$

$= \pm \frac{\sqrt{(1 / r)(\Delta-q)(2 \Delta+q)} \cosh ((\theta / 2) \xi)}{\sqrt{-q+4 \Delta+(q+2 \Delta) \cosh (\theta \xi)}}$

$=\varphi_{h}^{ \pm}(\operatorname{see}(45))$, 


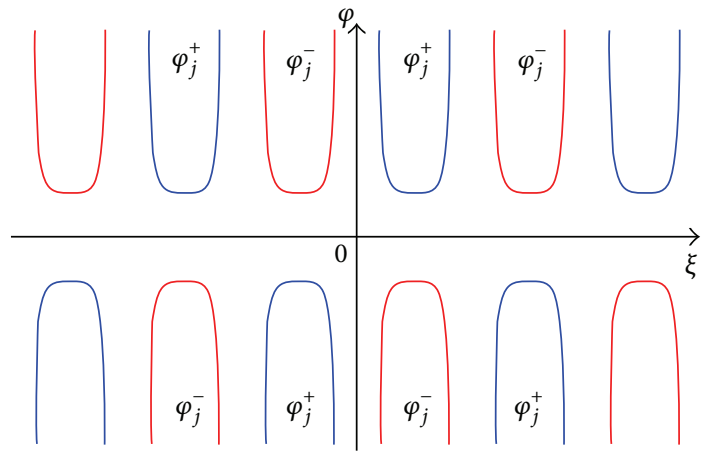

(a)

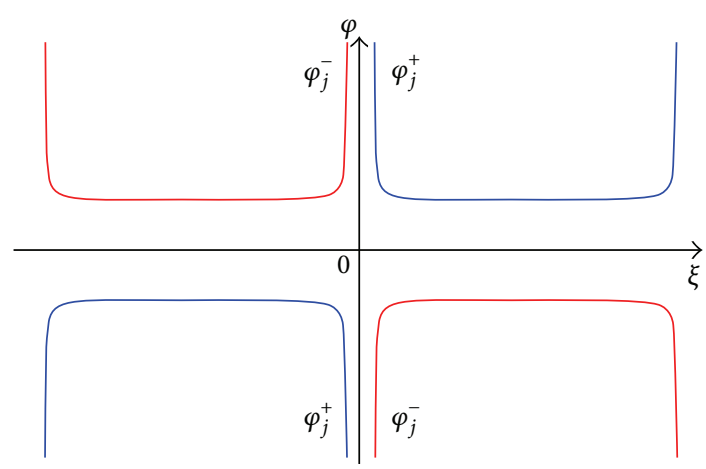

(c)

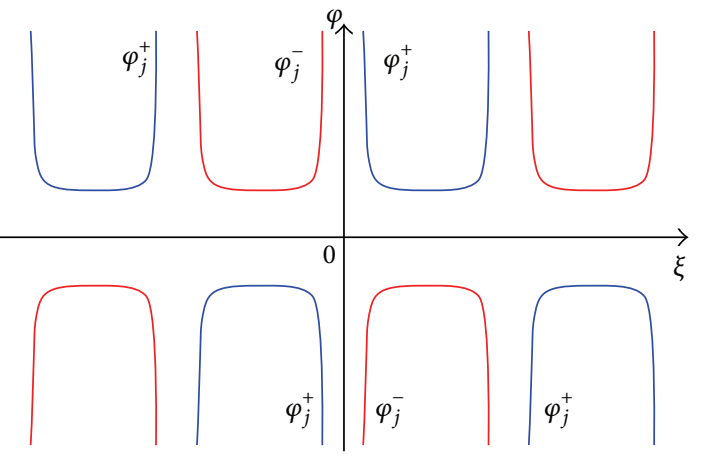

(b)

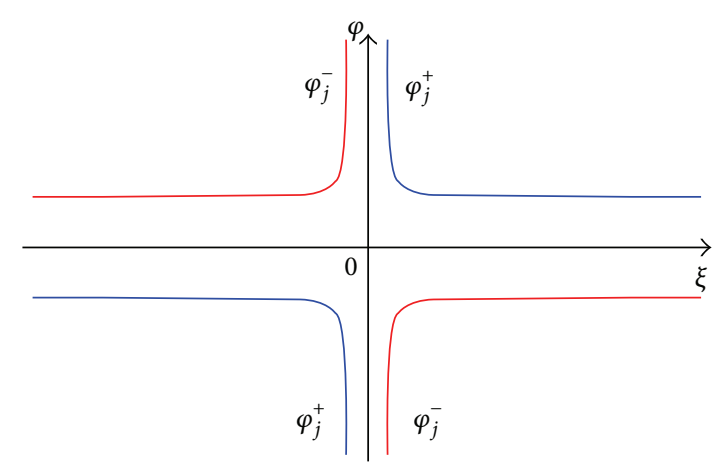

(d)

FIGURE 13: (The 1-blow-up waves are bifurcated from the periodic blow-up waves.) The varying process for the figures of $\varphi_{j}^{ \pm}$when $(q, p) \in A_{4}$ and $p \rightarrow q^{2} / 4 r-0$, where $r=1, q=-4$, and $l_{1}: p=q^{2} / 4 r=4$ and (a) $p=4-10^{-1}$, (b) $p=4-10^{-2}$, (c) $p=4-10^{-4}$, and (d) $p=4-10^{-6}$.

$\varphi_{f}^{ \pm}$

$$
\begin{aligned}
& = \pm \frac{\sqrt{(\Delta-q) / 2 r}\left(2 \Delta+q-2 \mu r e^{-\theta \xi}\right)}{\sqrt{(2 \Delta+q)^{2}+4 \mu r(4 \Delta-q) e^{-\theta \xi}+4 \mu^{2} r^{2} e^{-2 \theta \xi}}} \\
& = \pm \frac{\sqrt{(\Delta-q) / 2 r}(2 \Delta+q)\left(1+e^{-\theta \xi}\right)}{\sqrt{(q+2 \Delta)^{2}+2(q+2 \Delta)(4 \Delta-q) e^{-\theta \xi}+(q+2 \Delta)^{2} e^{-2 \theta \xi}}} \\
& = \pm \frac{\sqrt{(1 / 2 r)(\Delta-q)(2 \Delta+q)}\left(1+e^{-\theta \xi}\right)}{\sqrt{q+2 \Delta+2(4 \Delta-q) e^{-\theta \xi}+(q+2 \Delta) e^{-2 \theta \xi}}} \\
& = \pm \frac{\sqrt{(1 / 2 r)(\Delta-q)(2 \Delta+q)}\left(e^{-\theta \xi / 2}+e^{\theta \xi / 2}\right)}{\sqrt{2(4 \Delta-q)+(q+2 \Delta)\left(e^{-\theta \xi}+e^{\theta \xi}\right)}} \\
& = \pm \frac{\sqrt{(1 / r)(\Delta-q)(2 \Delta+q)} \cosh ((\theta / 2) \xi)}{\sqrt{-q+4 \Delta+(q+2 \Delta) \cosh (\theta \xi)}} \\
& =\varphi_{h}^{ \pm}(\operatorname{see}(45)) .
\end{aligned}
$$

For the case of $\mu<0$, similarly we can obtain the relations of the solutions $\varphi_{e}^{ \pm}, \varphi_{f}^{ \pm}, \varphi_{g}^{ \pm}$, and $\varphi_{h}^{ \pm}$, and here we omit the process. Hereto, we have completed the derivations for Proposition 2.

3.3. The Derivations to Proposition 3. When the orbit $\Gamma$ is defined by $H(\varphi, y)=H\left(\varphi_{2}, 0\right)$, firstly, if $(q, p)$ belongs to one of $A_{3}, A_{4}, A_{11}$, and $l_{2}$, then from (55) we obtain

$$
\begin{aligned}
y & = \pm \sqrt{p \varphi^{2}+\frac{q}{2} \varphi^{4}+\frac{r}{3} \varphi^{6}+H\left(\varphi_{2}, 0\right)} \\
& = \pm \sqrt{\frac{r}{3}\left(\varphi^{2}-\varphi_{2}^{2}\right)^{2}\left(\varphi^{2}+\delta_{0}\right)},
\end{aligned}
$$

where $\varphi_{2}$ is given in (16) and $\delta_{0}=(q-2 \Delta) / 2 r$. Substituting (75) into the first equation of (54) and integrating it, we have

$$
\int_{n}^{\varphi} \frac{d s}{\sqrt{(r / 3)\left(s^{2}-\varphi_{2}^{2}\right)^{2}\left(s^{2}+\delta_{0}\right)}}=\xi
$$

where $n$ is an arbitrary constant or $\pm \infty$.

Completing the above integral and solving for $\varphi$, it follows that

$$
\varphi= \pm \sqrt{\varphi_{2}^{2}-\frac{2 a_{2}}{b_{2}+\delta_{0} \sin (\delta+\eta \xi)}},
$$




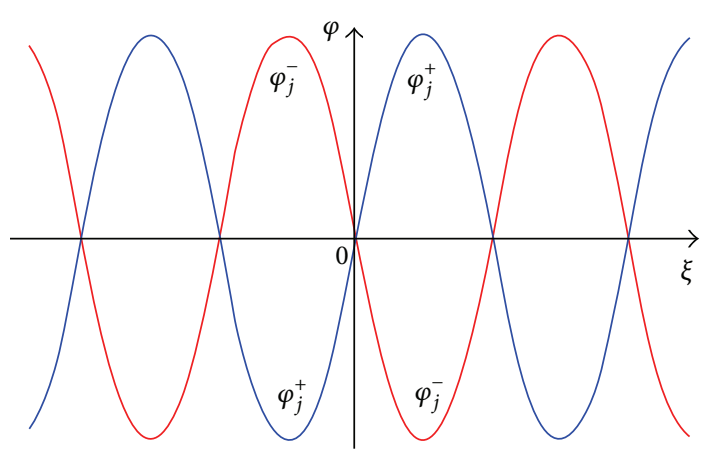

(a)

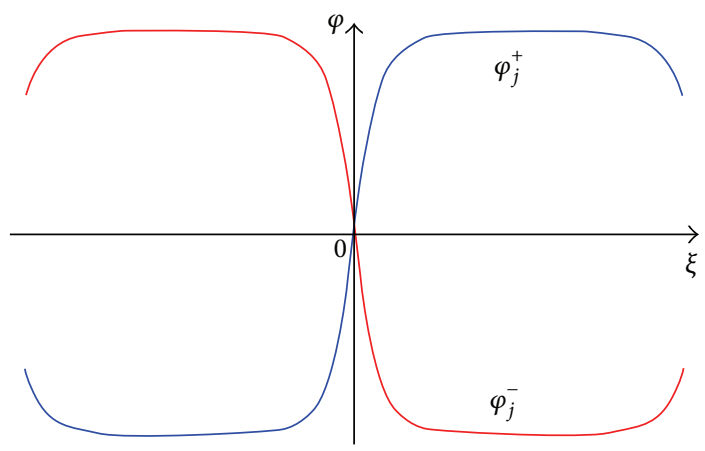

(c)

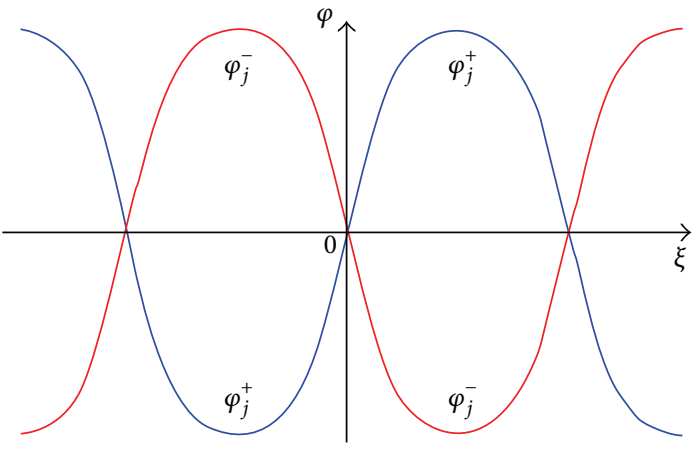

(b)

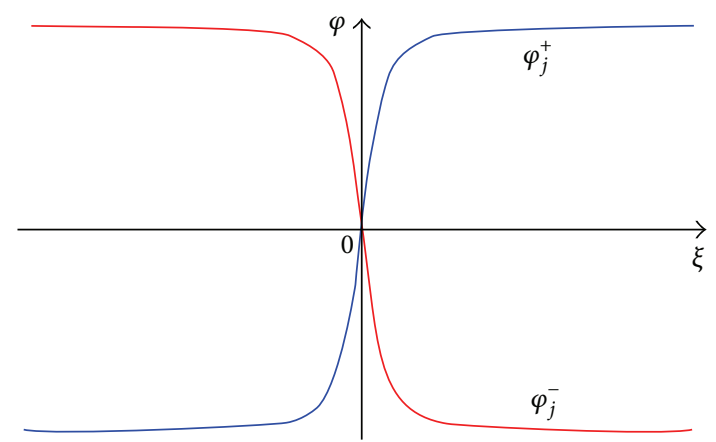

(d)

FIGURE 14: (The two tall-kink waves are bifurcated form two periodic waves.) The varying process for the figures of $\varphi_{j}^{ \pm}$when $(q, p) \in A_{11}$ and $p \rightarrow q^{2} / 4 r+0$, where $r=-1, q=4$, and $l_{7}: p=q^{2} / 4 r=-4$ and (a) $p=-3.5$, (b) $p=-4+10^{-1}$, (c) $p=-4+10^{-3}$, and (d) $p=-4+10^{-5}$.

where $\eta$ is given in (49), $\delta=\delta(n)$ is an arbitrary constant, and

$$
a_{2}=\frac{3 \Delta(\Delta+q)}{4 r^{2}}, \quad b_{2}=-\frac{q+4 \Delta}{2 r} \text {. }
$$

In (77) letting $\delta= \pm(\pi / 2)$, respectively, we obtain the solutions $\varphi_{i}^{ \pm}$and $\varphi_{j}^{ \pm}$as (47) and (48).

Secondly, if $(q, p) \in l_{1}$ or $l_{7}$, that is, $\Delta=0$ and $\varphi_{1}^{2}=-\delta_{0}=$ $-(q / 2 r)$, thus from (75), we obtain

$$
y= \pm \sqrt{\frac{r}{3}\left(\varphi^{2}+\frac{q}{2 r}\right)^{3}} .
$$

Similarly, we have

$$
\varphi= \pm \sqrt{\frac{-q}{2 r}} \frac{q \xi}{\sqrt{q^{2} \xi^{2}-12 r}}
$$

which yields $\varphi_{k}^{ \pm}$as in (51).
When $p \rightarrow q^{2} / 4 r$, it follows that

$$
\begin{gathered}
\Delta=\sqrt{q^{2}-4 p r} \longrightarrow 0, \\
\eta=\sqrt{\frac{1}{r}(4 p r-q(q+\Delta))}=\sqrt{-\frac{\Delta(\Delta+q)}{r}} \longrightarrow 0 \\
\cos (\eta \xi)=1-\frac{\eta^{2} \xi^{2}}{2}+\frac{\eta^{4} \xi^{4}}{4 !}+\cdots \\
=1+\frac{\Delta(\Delta+q)}{2 r} \xi^{2}+o\left(\Delta^{2}\right) .
\end{gathered}
$$

Thus, we have

$$
\begin{aligned}
& \lim _{p \rightarrow\left(q^{2} / 4 r\right)} \varphi_{i}^{ \pm} \\
& \quad= \pm \lim _{p \rightarrow q^{2} / 4 r} \sqrt{\frac{(q+\Delta)(2 \Delta-q)}{r(q+4 \Delta+(q-2 \Delta) \cos (\eta \xi))}} \cos \left(\frac{\eta}{2} \xi\right) \\
& = \pm \sqrt{\frac{-q^{2}}{2 r q}}= \pm \sqrt{-\frac{q}{2 r}},
\end{aligned}
$$




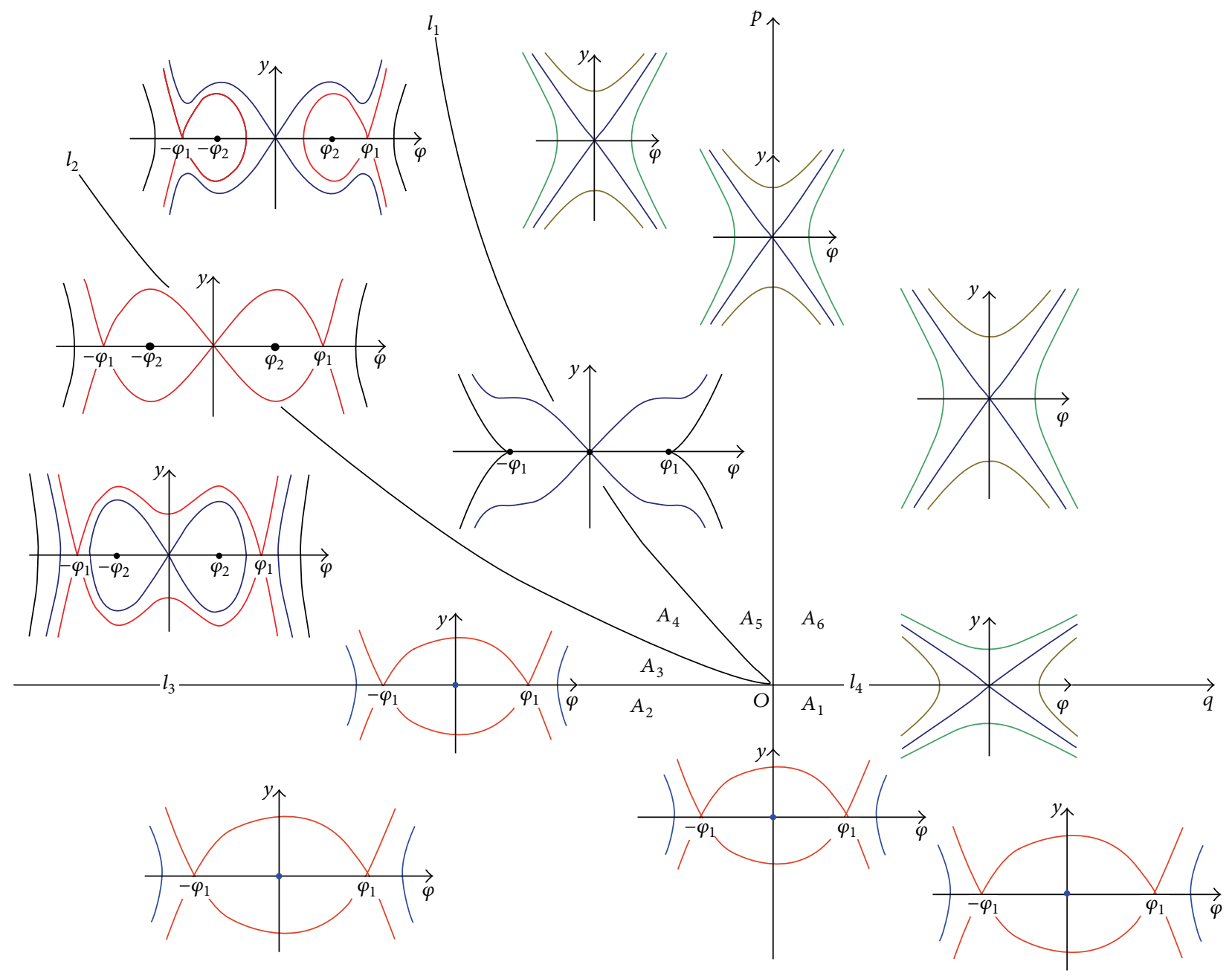

FIGURE 15: The bifurcation phase portraits of system (54) for $r>0$.

$$
\begin{array}{r}
\lim _{p \rightarrow q^{2} / 4 r} \varphi_{j}^{ \pm} \\
=\lim _{p \rightarrow q^{2} / 4 r} \pm \sqrt{\frac{(q+\Delta)(2 \Delta-q)}{r(q+4 \Delta-(q-2 \Delta) \cos (\eta \xi))}} \sin \left(\frac{\eta}{2} \xi\right) \\
=\lim _{p \rightarrow q^{2} / 4 r} \pm \sqrt{\frac{(q+\Delta)(2 \Delta-q)(1-\cos (\eta \xi))}{2 r(q+4 \Delta-(q-2 \Delta) \cos (\eta \xi))}} \\
=\lim _{p \rightarrow q^{2} / 4 r} \pm\left(\begin{array}{l}
-(q+\Delta)(2 \Delta-q) \frac{\Delta(\Delta+q)}{2 r} \xi^{2} \\
\left.+o(\Delta)^{2}\right) \times(2 r[q+4 \Delta-(q-2 \Delta) \\
\times\left(1+\frac{\Delta(\Delta+q)}{2 r} \xi^{2}\right) \\
\left.\left.\left.+o\left(\Delta^{2}\right)\right]\right)^{-1}\right)^{1 / 2}
\end{array}\right.
\end{array}
$$

$$
\begin{aligned}
& =\lim _{p \rightarrow q^{2} / 4 r} \pm\left(\left(-\frac{(\Delta+q)^{2}(2 \Delta-q)}{2 r} \xi^{2}+o(\Delta)\right)\right. \\
& \quad \times\left(2 r \left[6-(q-2 \Delta)\left(1+\frac{\Delta(\Delta+q)}{2 r} \xi^{2}\right)\right.\right. \\
& \left.+o(\Delta)])^{-1}\right)^{1 / 2} \\
& = \pm \sqrt{\frac{\left(q^{3} / 2 r\right) \xi^{2}}{2 r\left(6-\left(q^{2} / 2 r\right) \xi^{2}\right)}} \\
& = \pm \sqrt{\frac{-q}{2 r} \frac{q \xi}{\sqrt{q^{2} \xi^{2}-12 r}}} \\
& =\varphi_{k}^{ \pm}(\operatorname{see}(51)) .
\end{aligned}
$$

Hereto, we have completed the derivations for our main results. 


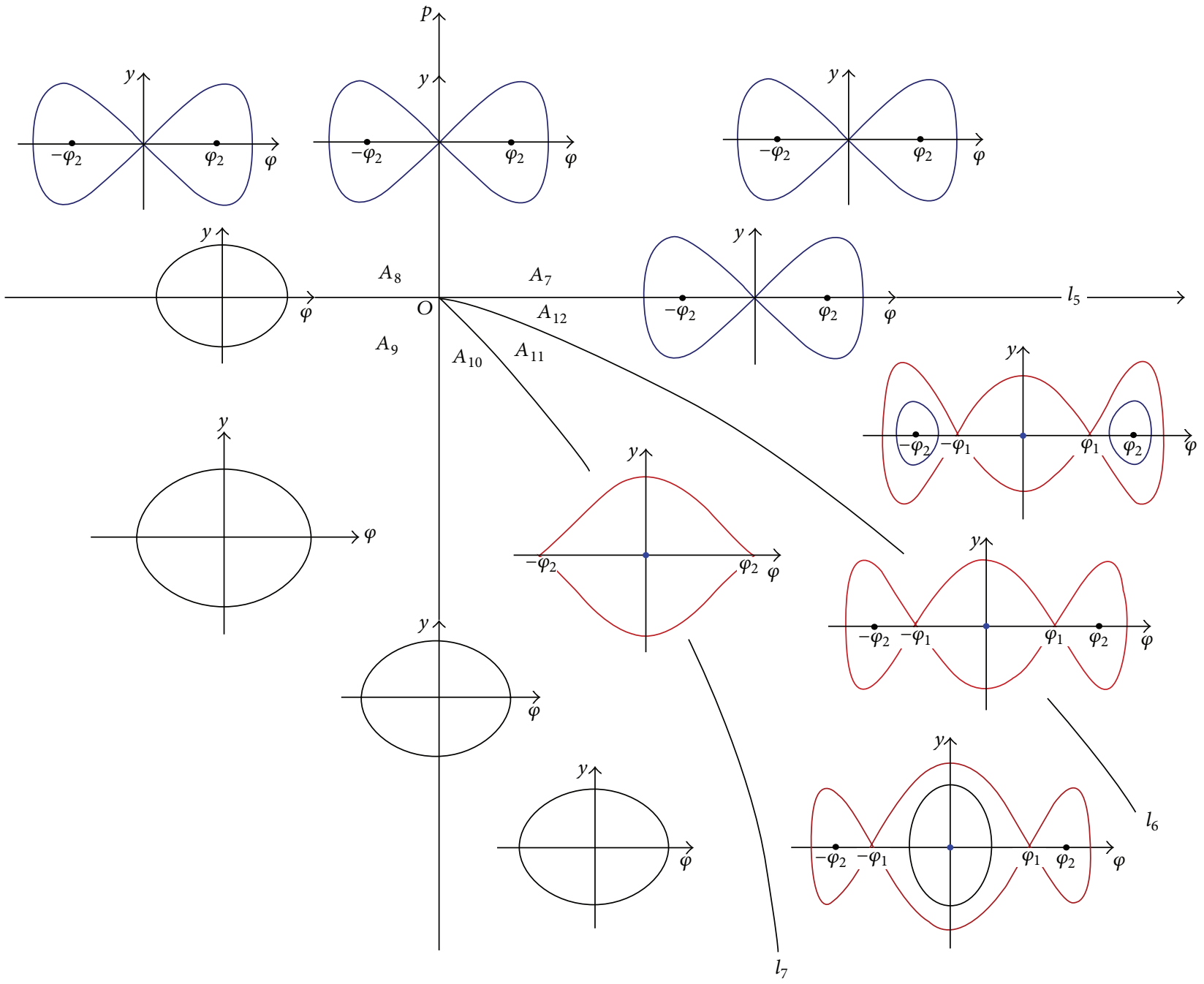

FIGURE 16: The bifurcation phase portraits of system (54) for $r<0$.

\section{Conclusions}

In this paper, we have investigated the explicit expressions of the nonlinear waves and bifurcation phenomena in (1). Firstly, we obtained three types of explicit nonlinear wave solutions. The first type is the fractional expressions $\varphi_{a}^{ \pm}$and $\varphi_{k}^{ \pm}$(see (29) and (51)). The second type is the trigonometric expressions $\varphi_{i}^{ \pm}$and $\varphi_{j}^{ \pm}$(see (47) and (48)). The third type is the exp-function expressions $\varphi_{c}^{ \pm}, \varphi_{d}^{ \pm}, \varphi_{e}^{ \pm}$, and $\varphi_{f}^{ \pm}$(see (33) and (38)).

Secondly, we revealed five kinds of interesting bifurcation phenomena in (1). The first phenomena is that the 1-blowup waves can be bifurcated from the periodic-blow-up (see Figure 2 or Figure 13) and 2-blow-up waves (see Figure 8). The second phenomena is that the 2-blow-up waves can be bifurcated from the periodic-blow-up waves (see Figure 3). The third kind is that the symmetric solitary waves can be bifurcated from the symmetric periodic waves (see Figure 4). The fourth kind is that the low-kink waves can be bifurcated from the symmetric solitary waves (see Figure 6), the 1-blowup waves (see Figure 7), the tall-kink waves (see Figure 9), and the antisymmetric solitary waves (see Figure 10). The fifth kind is that the tall-kink waves can be bifurcated from the symmetric periodic waves (see Figure 14).

Thirdly, we showed that some previous results are our special cases. For instants, $\varphi_{b 0}^{ \pm}, \varphi_{c 0}^{ \pm}$, and $\varphi_{e 0}^{ \pm}$are included in $\varphi_{c}^{ \pm}, \varphi_{d}^{ \pm}, \varphi_{e}^{ \pm}$, and $\varphi_{f}^{ \pm}$(see (4), (5), (7), (33), and (38)). $\varphi_{d 0}^{ \pm}$are included in $\varphi_{c}^{ \pm}$and $\varphi_{d}^{ \pm}$(see (6) and (33)).

Finally, we employed the software Mathematica to check the correctness of these solutions. For example, the commands for $u_{a}$ and $E_{a}$ are as follows:

$$
\begin{aligned}
& w=\alpha \gamma^{2}, \\
& p=\left(\alpha \gamma^{2}-w\right) / \alpha, \\
& q=\beta \delta_{1} / \alpha\left(c^{2}-c_{s}^{2}\right)-\delta_{2} / \alpha, \\
& r=-\delta_{3} / \alpha, \\
& c=2 \alpha \gamma, \\
& \xi=x-c t, \\
& \varphi_{a}=\sqrt{6 q /\left(3 q^{2} \xi^{2}-4 r\right)},
\end{aligned}
$$




$$
\begin{aligned}
& u_{a}=\left(\beta /\left(c^{2}-c_{s}^{2}\right)\right) \varphi_{a}^{2}, \\
& E_{a}=\varphi_{a} \operatorname{Exp}[i(\gamma x-w t)], \\
& \text { Simplify }\left[\mathrm{D}\left[u_{a},\{t, 2\}\right]-c_{s}^{2} \mathrm{D}\left[u_{a},\{x, 2\}\right]-\beta \mathrm{D}\left[\varphi_{a}^{2},\{x, 2\}\right]\right] \\
& \text { Simplify }\left[i \mathrm{D}\left[E_{a}, t\right]+\alpha \mathrm{D}\left[E_{a},\{x, 2\}\right]-\delta_{1} u_{a} E_{a}+\right. \\
& \left.\delta_{2} \varphi_{a}^{2} E_{a}+\delta_{3} \varphi_{a}^{4} E_{a}\right] \\
& 0 \\
& 0
\end{aligned}
$$

\section{Acknowledgment}

This paper is supported by the National Natural Science Foundation (no. 11171115), Natural Science Foundation of Guangdong Province (no. S2012040007959) and the Fundamental Research Funds for the Central Universities (no. 2012ZM0057).

\section{References}

[1] G.-T. Liu and T.-Y. Fan, "New applications of developed Jacobi elliptic function expansion methods," Physics Letters A, vol. 345, no. 1-3, pp. 161-166, 2005.

[2] S. Liu, Z. Fu, S. Liu, and Q. Zhao, "Jacobi elliptic function expansion method and periodic wave solutions of nonlinear wave equations," Physics Letters A, vol. 289, no. 1-2, pp. 69-74, 2001.

[3] M. A. Abdou, "The extended F-expansion method and its application for a class of nonlinear evolution equations," Chaos, Solitons \& Fractals, vol. 31, no. 1, pp. 95-104, 2007.

[4] M. Wang and X. Li, "Extended F-expansion method and periodic wave solutions for the generalized Zakharov equations," Physics Letters. A, vol. 343, no. 1-3, pp. 48-54, 2005.

[5] M. Wang, X. Li, and J. Zhang, “The $G^{\prime} / G$-expansion method and travelling wave solutions of nonlinear evolution equations in mathematical physics," Physics Letters A, vol. 372, no. 4, pp. 417-423, 2008.

[6] M. Song and Y. Ge, "Application of the $G^{\prime} / G$-expansion method to (3+1)-dimensional nonlinear evolution equations," Computers \& Mathematics with Applications, vol. 60, no. 5, pp. 12201227, 2010.

[7] J. B. Li and Z. R. Liu, "Smooth and non-smooth traveling waves in a nonlinearly dispersive equation," Applied Mathematical Modelling, vol. 25, pp. 41-56, 2000.

[8] Z. Liu and J. Li, "Bifurcations of solitary waves and domain wall waves for KdV-like equation with higher order nonlinearity," International Journal of Bifurcation and Chaos in Applied Sciences and Engineering, vol. 12, no. 2, pp. 397-407, 2002.

[9] Z. R. Liu and C. X. Yang, "The application of bifurcation method to a higher-order KdV equation," Journal of Mathematical Analysis and Applications, vol. 275, no. 1, pp. 1-12, 2002.

[10] Z. R. Liu and Y. Liang, "The explicit nonlinear wave solutions and their bifurcations of the generalized Camassa-Holm equation," International Journal of Bifurcation and Chaos in Applied Sciences and Engineering, vol. 21, no. 11, pp. 3119-3136, 2011.

[11] R. Liu, "Some new results on explicit traveling wave solutions of $K(m, n)$ equation," Discrete and Continuous Dynamical Systems $B$, vol. 13, no. 3, pp. 633-646, 2010.

[12] R. Liu, "Coexistence of multifarious exact nonlinear wave solutions for generalized b-equation," International Journal of
Bifurcation and Chaos in Applied Sciences and Engineering, vol. 20, no. 10, pp. 3193-3208, 2010.

[13] M. Song, S. Li, and J. Cao, "New exact solutions for the (2+1)dimensional Broer-Kaup-Kupershmidt equations," Abstract and Applied Analysis, vol. 2010, Article ID 652649, 9 pages, 2010.

[14] M. Song, X. Hou, and J. Cao, "Solitary wave solutions and kink wave solutions for a generalized KDV-mKDV equation," Applied Mathematics and Computation, vol. 217, no. 12, pp. 5942-5948, 2011.

[15] M. Song, J. Cao, and X. Guan, "Application of the bifurcation method to the Whitham-Broer-Kaup-like equations," Mathematical and Computer Modelling, vol. 55, no. 3-4, pp. 688-696, 2012.

[16] D. Feng and J. Li, "Exact explicit travelling wave solutions for the $(n+1)$-dimensional $\phi^{6}$ field model," Physics Letters A, vol. 369, no. 4, pp. 255-261, 2007.

[17] D.-J. Huang and H.-Q. Zhang, "New exact travelling waves solutions to the combined KdV-MKdV and generalized Zakharov equations," Reports on Mathematical Physics, vol. 57, no. 2, pp. 257-269, 2006.

[18] S. A. El-Wakil, M. A. Abdou, and A. Elhanbaly, "New solitons and periodic wave solutions for nonlinear evolution equations," Physics Letters A, vol. 353, pp. 40-47, 2006.

[19] J. Wang, "Multisymplectic numerical method for the Zakharov system," Computer Physics Communications, vol. 180, no. 7, pp. 1063-1071, 2009.

[20] B. J. Hong, D. C. Lu, and F. S. Sun, "The extended Jacobi Elliptic Functions expansion method and new exact solutions for the Zakharov equations," World Journal of Modelling and Simulation, vol. 5, pp. 216-224, 2009.

[21] O. P. Layeni, "A new rational auxiliary equation method and exact solutions of a generalized Zakharov system," Applied Mathematics and Computation, vol. 215, no. 8, pp. 2901-2907, 2009.

[22] Y. Xia, Y. Xu, and C.-W. Shu, "Local discontinuous Galerkin methods for the generalized Zakharov system," Journal of Computational Physics, vol. 229, no. 4, pp. 1238-1259, 2010.

[23] G. Betchewe, B. B. Thomas, K. K. Victor, and K. T. Crepin, "Dynamical survey of a generalized-Zakharov equation and its exact travelling wave solutions," Applied Mathematics and Computation, vol. 217, no. 1, pp. 203-211, 2010.

[24] A. Bekir and A. C. Cevikel, "New solitons and periodic solutions for nonlinear physical models in mathematical physics," Nonlinear Analysis. Real World Applications, vol. 11, no. 4, pp. 3275-3285, 2010. 


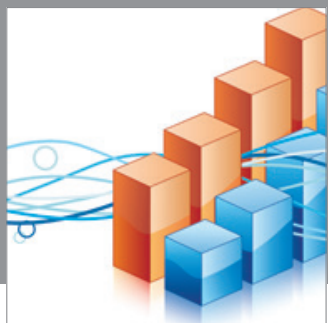

Advances in

Operations Research

mansans

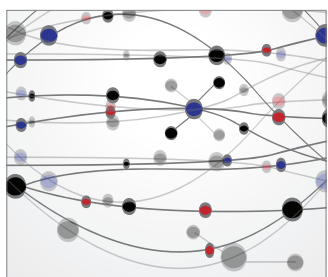

The Scientific World Journal
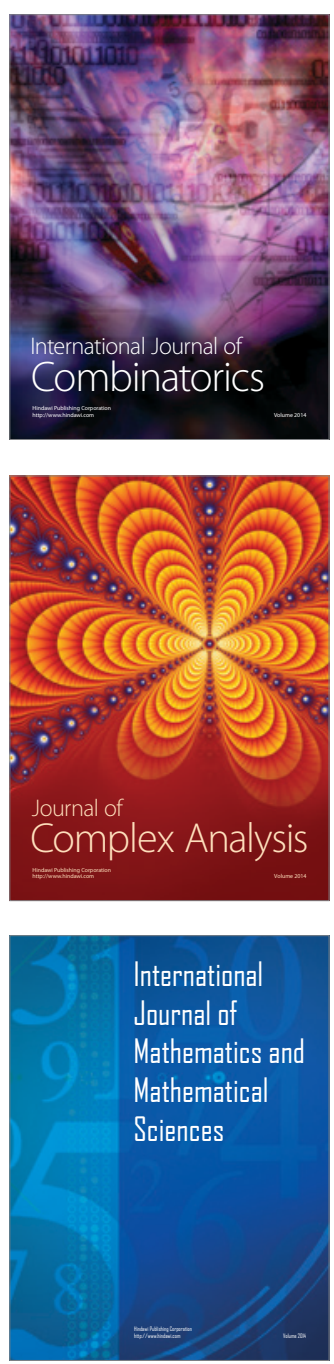
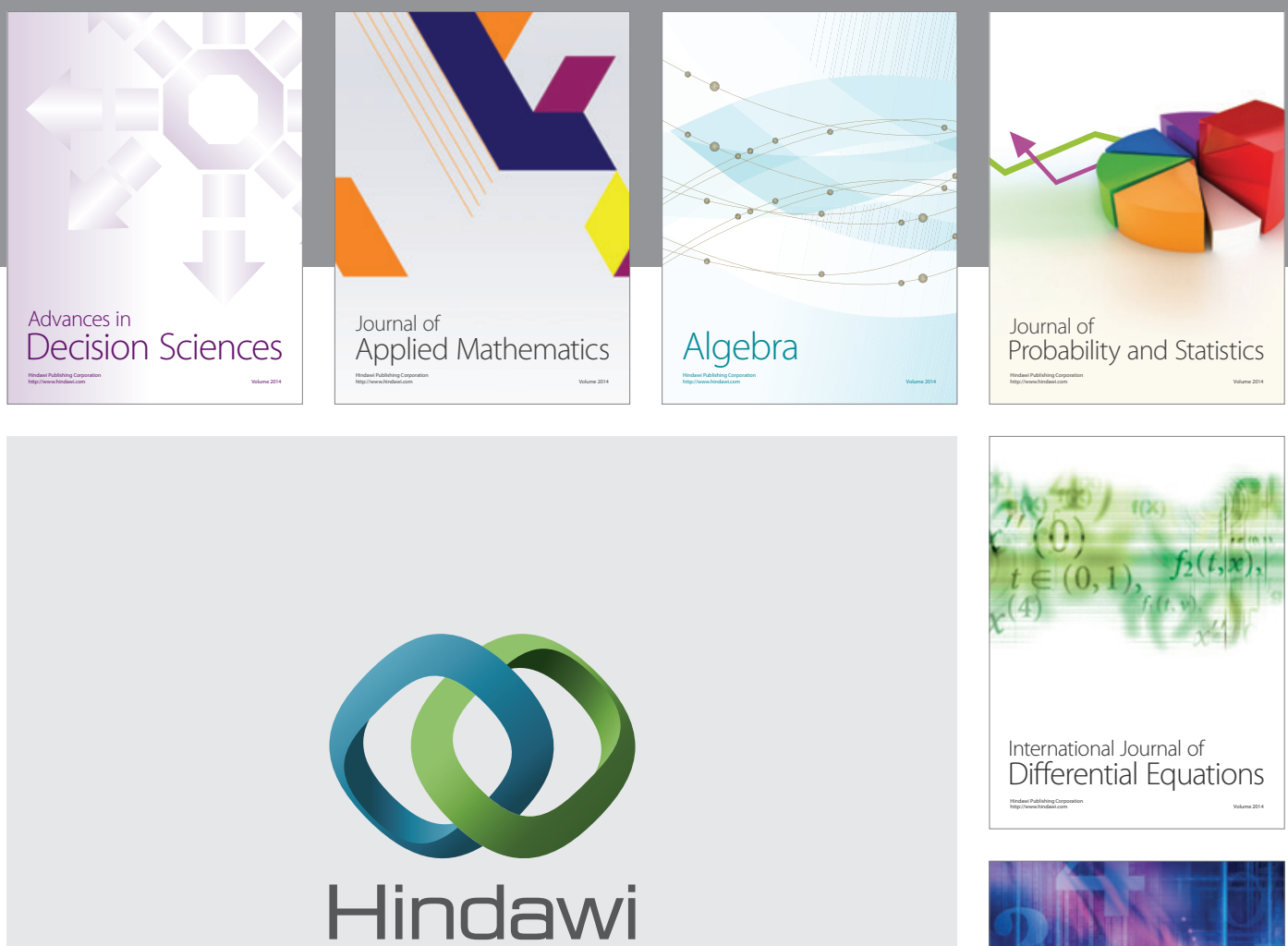

Submit your manuscripts at http://www.hindawi.com
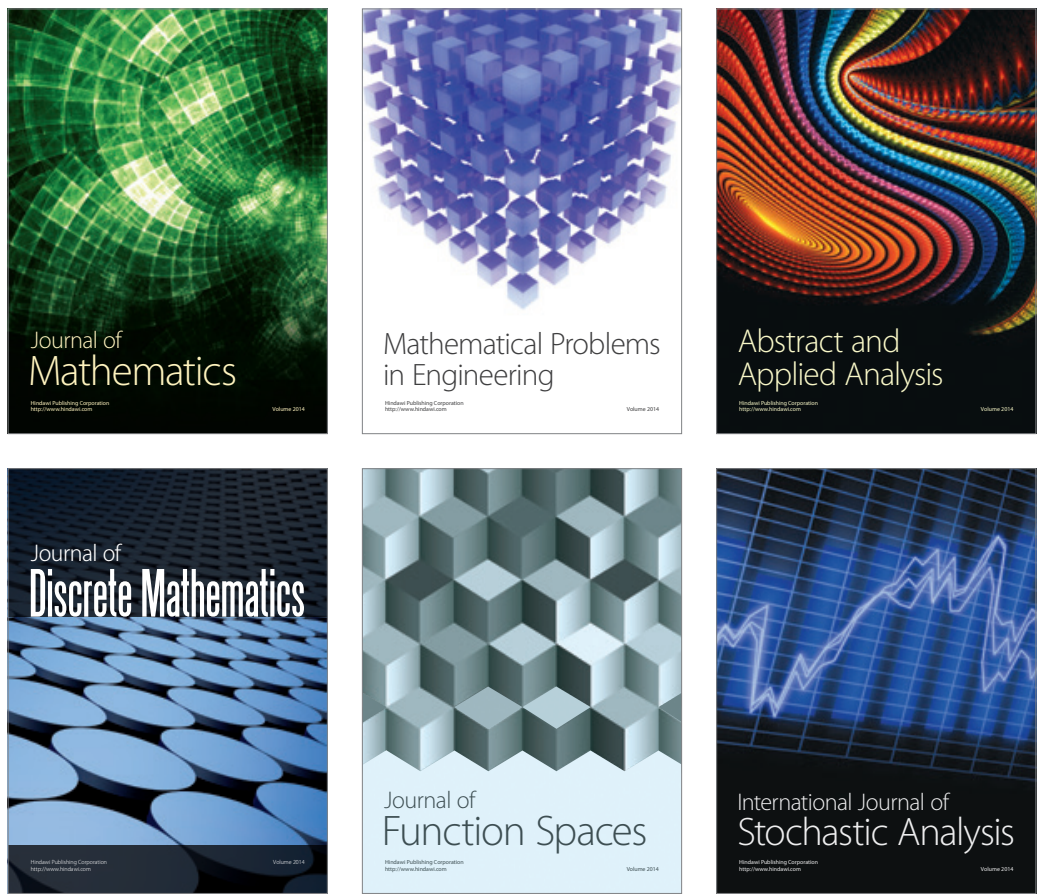

Journal of

Function Spaces

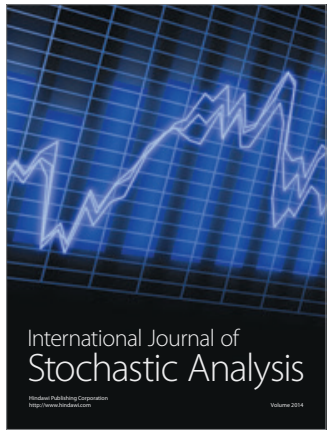

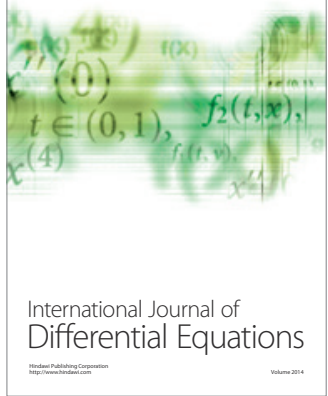
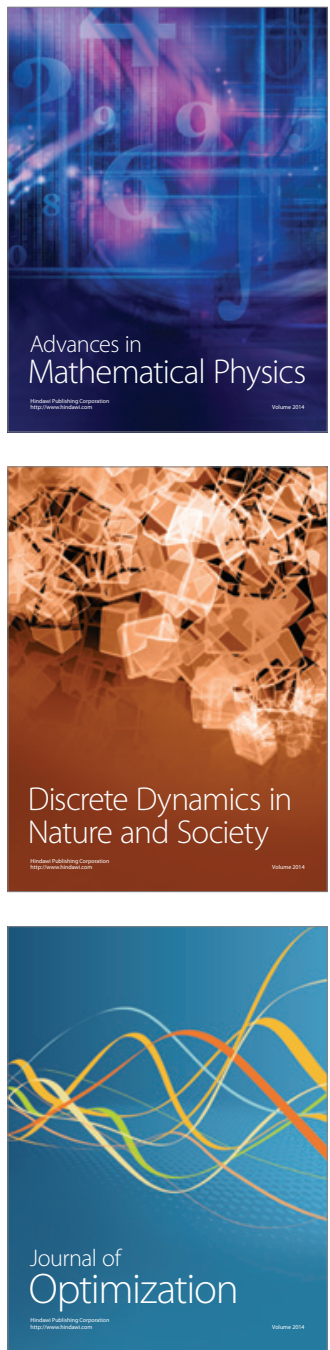